\title{
Sphingolipid-mediated inflammatory signaling leading to autophagy inhibition converts erythropoiesis to myelopoiesis in human hematopoietic stem/progenitor cells
}

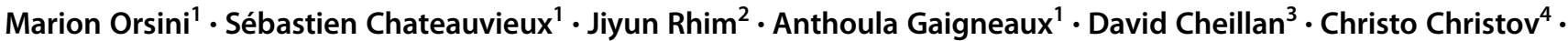 \\ Mario Dicato ${ }^{1} \cdot$ Franck Morceau $^{1} \cdot$ Marc Diederich $^{2}$
}

Received: 15 August 2017 / Revised: 4 November 2018 / Accepted: 19 November 2018 / Published online: 13 December 2018

(c) The Author(s) 2018. This article is published with open access

\begin{abstract}
Elevated levels of the pro-inflammatory cytokine tumor necrosis factor- $\alpha(\mathrm{TNF} \alpha)$ inhibit erythropoiesis and cause anemia in patients with cancer and chronic inflammatory diseases. TNF $\alpha$ is also a potent activator of the sphingomyelinase (SMase)/ ceramide pathway leading to ceramide synthesis and regulating cell differentiation, proliferation, apoptosis, senescence, and

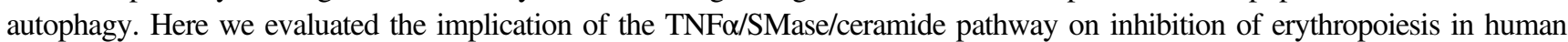
$\mathrm{CD}^{+}{ }^{+}$hematopoietic stem/progenitor cells (CD34/HSPCs) from healthy donors. Exogenous synthetic C2- and C6-ceramide as well as bacterial SMase inhibited erythroid differentiation in erythropoietin-induced (Epo)CD34/HSPCs shown by the analysis of various erythroid markers. The neutral SMase inhibitor GW4869 as well as the genetic inhibition of nSMase with small interfering RNA (siRNA) against sphingomyelin phosphodiesterase 3 (SMPD3) prevented the inhibition by TNF $\alpha$, but not the acid SMase inhibitor desipramine. Moreover, sphingosine-1-phosphate (S1P), a ceramide metabolite, restored erythroid differentiation, whereas TNFo inhibited sphingosine kinase-1, required for S1P synthesis. Analysis of cell morphology and colony formation demonstrated that erythropoiesis impairment was concomitant with a granulomonocytic differentiation in TNF $\alpha$ - and ceramidetreated EpoCD34/HSPCs. Inhibition of erythropoiesis and induction of granulomonocytic differentiation were correlated to modulation of hematopoietic transcription factors (TFs) GATA-1, GATA-2, and PU.1. Moreover, the expression of microRNAs (miR)-144/451, miR-146a, miR-155, and miR-223 was also modulated by TNF $\alpha$ and ceramide treatments, in line with cellular observations. Autophagy plays an essential role during erythropoiesis and our results demonstrate that the TNF $\alpha /$ neutral SMase/ ceramide pathway inhibits autophagy in EpoCD34/HSPCs. TNF $\alpha$ - and ceramide-induced phosphorylation of $\mathrm{mTOR}^{\mathrm{S} 2448}$ and $\mathrm{ULK} 1^{\mathrm{S} 758}$, inhibited Atg1 $3^{\mathrm{S} 355}$ phosphorylation, and blocked autophagosome formation as shown by transmission electron microscopy and GFP-LC3 punctae formation. Moreover, rapamycin prevented the inhibitory effect of TNF $\alpha$ and ceramides on erythropoiesis while inhibiting induction of myelopoiesis. In contrast, bafilomycin A1, but not siRNA against Atg5, induced myeloid differentiation, while both impaired erythropoiesis. We demonstrate here that the TNF $\alpha /$ neutral SMase/ceramide pathway inhibits erythropoiesis to induce myelopoiesis via modulation of a hematopoietic TF/miR network and inhibition of late steps of autophagy. Altogether, our results reveal an essential role of autophagy in erythroid vs. myeloid differentiation.
\end{abstract}

Edited by J.P. Medema

Supplementary information The online version of this article (https:// doi.org/10.1038/s41418-018-0245-x) contains supplementary material, which is available to authorized users.

Marc Diederich

marcdiederich@snu.ac.kr

1 Laboratoire de Biologie Moléculaire et Cellulaire du Cancer, Hôpital Kirchberg, 9, rue Edward Steichen, 2540 Luxembourg, Luxembourg

2 Department of Pharmacy, Research Institute of Pharmaceutical Sciences, College of Pharmacy, Seoul National University, 1 Gwanak-ro, Gwanak-gu, Seoul 08826, South Korea
3 Service de Biochimie et Biologie Moléculaire Grand Est, Unité Médicale Pathologies Métaboliques Erythrocytaires et Dépistage Périnatal, Centre de Biologie et de Pathologie Est, Hospices Civils de Lyon, Bron, France

4 Service Commun de Microscopie, Université de Lorraine, Nancy, France 


\section{Introduction}

Inflammatory cytokines cause anemia of cancer and chronic inflammatory diseases with consequences for the quality of life and lifespan. Cytokines like tumor necrosis factor- $\alpha$ (TNF $\alpha)$ impair erythroid differentiation and perturbate hematopoietic homeostasis [1-5]. A network of receptors, cell signaling cascades, transcription factors (TFs), and microRNAs (miRs) time-dependently regulate hematopoiesis $[6,7]$. TFs and miRs modulate genes involved in myeloid commitment. Major TF GATA-1 regulates erythropoiesis by modulating the negative regulator GATA-2 [8] and the erythroid miR-144/451 gene cluster [9]. Conversely, myeloid regulator PU.1 prevents erythropoiesis by binding to GATA-1. The GATA-1/PU.1 antagonism determines erythroid vs. myeloid cell fate [10]. Furthermore, PU.1 regulates miR-146a, miR-155, and miR-223 genes associated with inhibition of erythropoiesis and granulomonocytic development [11-13].

Erythrocytic but not granulomonocytic differentiation requires autophagic clearance of cytoplasmic organelles and expulsion of the nucleus [14-18]. Autophagosome formation requires the ULK1-Atg13-FIP200 complex [19]. This complex is inhibited by activation of the PI3K/AKT/ mTOR pathway, which prevents $\operatorname{Atg} 13^{\mathrm{S} 355}$ activation through ULK $1^{\text {S758 }}$ phosphorylation [20]. Vesicle nucleation involves Beclin-1 interacting with Rubicon (RUN domain protein as Beclin-1-interacting and cysteine-rich containing), a suppressor of autophagy [21]. Autophagosome formation requires Atg12/Atg5/Atg16 complex formation and lipidation of LC3 by Atg3/Atg7 [22]. Autophagosomes then fuse with endosomes/lysosomes triggering organelle and protein degradation including p62/ SQSTM1 cargo [23].

$\mathrm{TNF} \alpha$-stimulated nuclear factor- $\mathrm{KB}$ and $\mathrm{p} 38 \mathrm{MAPK}$ pathways regulate the inhibition of erythroid gene expression and differentiation $[4,24]$. TNF $\alpha$ also activates neutral sphingomyelinase (nSMase) through a nSMase activation domain within the cytoplasmic region of the $55 \mathrm{kDa} \mathrm{TNF}$ receptor. TNF receptor internalization also leads to acid (a) SMase activation [25]. SMases subsequently hydrolyze sphingomyelin to ceramides [26-28], which regulate inflammation, differentiation, proliferation, apoptosis, senescence, and autophagy [29, 30]. Ceramides are metabolized to sphingosine and sphingosine-1-phosphate (S1P) by ceramidase and sphingosine kinase-1 (SphK1). Ceramide and S1P play antagonistic roles in various cellular processes forming the "sphingolipid rheostat" [31].

As the regulatory role of ceramides remains unknown in $\mathrm{TNF} \alpha$-mediated impairment of erythropoiesis, we used here an erythropoiesis model based on $\mathrm{CD} 34^{+}$/hematopoietic stem progenitor cells (CD34/HSPCs) from umbilical cord blood (UCB) of healthy human donors. Altogether, we describe a novel $\mathrm{TNF} \alpha / \mathrm{nSMase} / \mathrm{ceramide}$ pathway that inhibits erythropoiesis and promotes myeloid differentiation by modulating a $\mathrm{TF} / \mathrm{miR}$ hematopoietic micro-network and by inhibiting autophagy.

\section{Results}

\section{The TNFa/nSMase/ceramide pathway downregulates erythroid differentiation}

We initially assessed the ability of TNF $\alpha$ and GW4869 to modulate ceramide synthesis in erythropoietin (Epo)-treated CD34 ${ }^{+} / \mathrm{HSPCs}$ (EpoCD34/HSPCs) by electrospray ionization-tandem mass spectrometry (ESI-MS/MS). Results showed that TNF $\alpha$ increased ceramide formation and particularly long-chain ceramide C24 and C24:1 after $24 \mathrm{~h}$ of treatment, while GW4869 prevented the effect of TNF $\alpha$ (Fig. 1a and Supplementary table S1). MS analysis and fluorescent immunostaining assays confirmed the rapid activation of ceramide production as early as $2 \mathrm{~min}$ (Supplementary Figure S1).

To study the implication of the sphingolipid metabolism in TNF $\alpha$-mediated inhibition of erythropoiesis, cell hemoglobinization was assessed by benzidine staining in EpoCD34/HSPCs. Results showed that TNF $\alpha$ inhibited hemoglobinization by $50 \%$ and that GW4869 treatment prevented this inhibition, whereas desipramine had no significant effect (Fig. 1b). Knock down of nSMase in EpoCD34/HSPCs by SMPD3-specific small interfering RNA (siRNA) (si-SMPD3) did not affect cell viability and allowed inhibition of nSMase messenger RNA (mRNA) and protein expression (Supplementary Figure S2). Results showed that the knock down of nSMase expression was able to restore the amount of hemoglobin-producing cells (Fig. 1c). The effect of ceramide production was then mimicked by treating EpoCD34/HSPCs with synthetic C2and C6-ceramide, bacterial SMase (bSMase), and the bioactive sphingolipids derived from ceramide C2dihydroceramide (dihydroceramide) and S1P. Like TNF $\alpha$, C2- and C6-ceramide as well as bSMase prevented hemoglobinization of EpoCD34/HSPCs by $40 \%$ at $96 \mathrm{~h}$, while dihydroceramide and S1P remained without effect (Fig. 1d and Supplementary Figure S3a). Nevertheless, S1P prevented $\mathrm{TNF} \alpha$-mediated inhibition of hemoglobinization, which was increased by $35 \%$. Moreover, the inhibition of S1P synthesis by SphK1 inhibitor PF-543 reduced hemoglobinization by $75 \%$ and enhanced the effect of $\mathrm{TNF} \alpha$ (Fig. 1e). Accordingly, TNF $\alpha$ inhibited SphK1 mRNA and protein expression, suggesting that inhibition of S1P production by TNF $\alpha$ contributed to impairment of erythropoiesis (Fig. 1f). Results showed that Epo induced three cell populations differentially expressing GPA: GPA $^{\text {low }}$, 
a

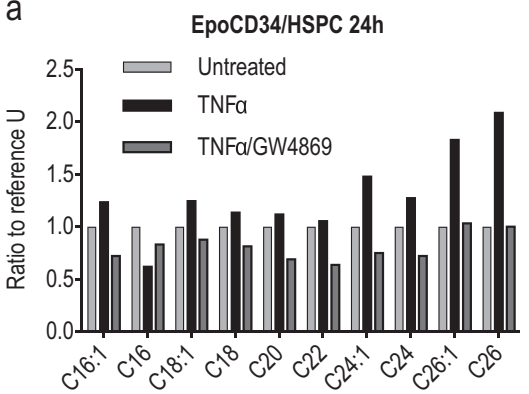

d

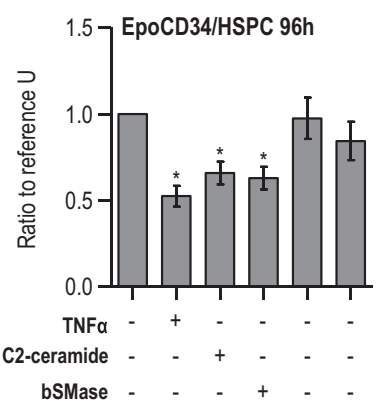

Dihydroceramide - - - - +

g
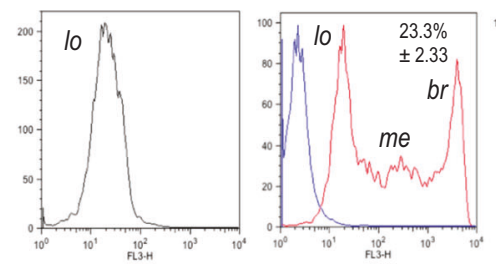

Untreated

CD34/HSPC Oh

h
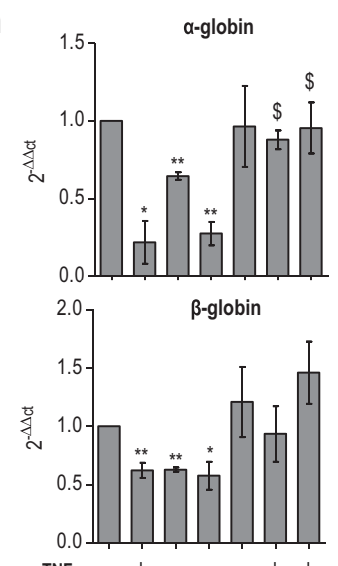

TNFa - + - - + +

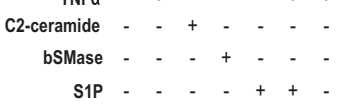

GW4869 - . . . . + b

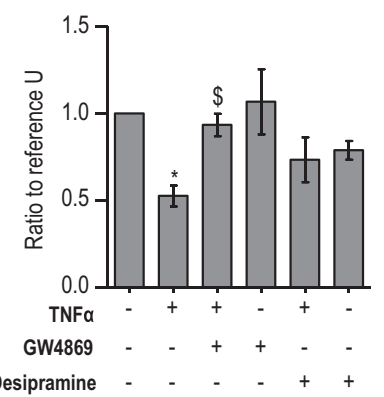

e

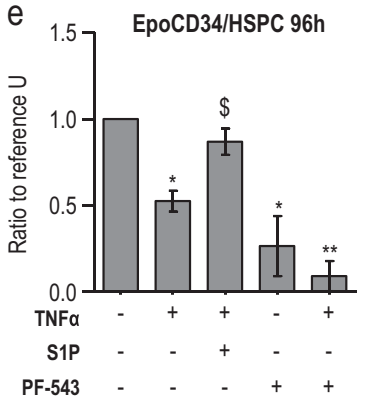

C EpoCD34/HSPC 96h

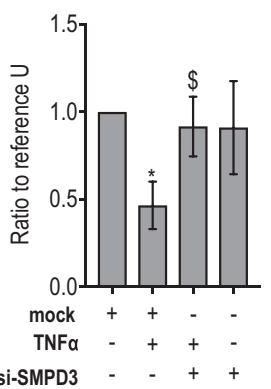

f

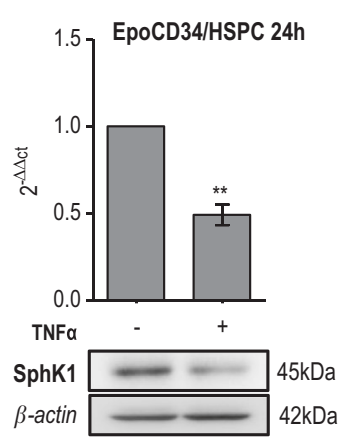

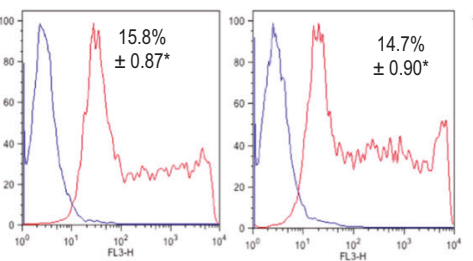

C2-ceramide

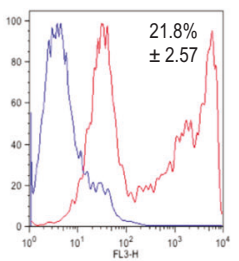

Dihydroceramide

EpoCD34/HSPC 96h

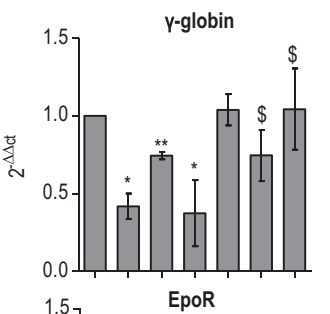

i

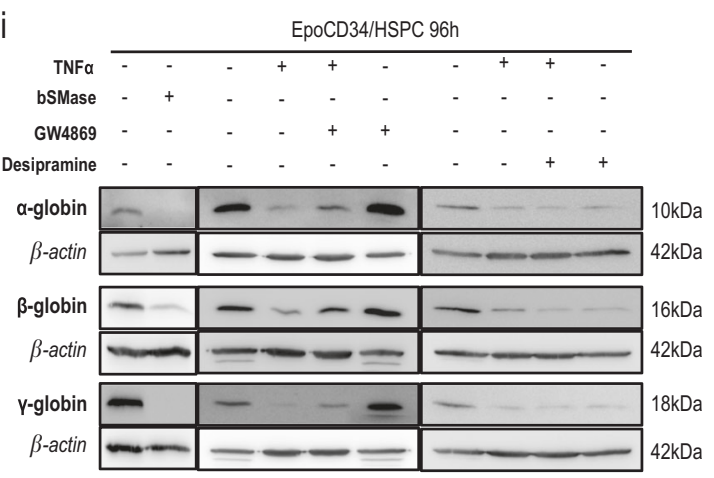

GPA $^{\text {medium }}$, and GPA ${ }^{\text {bright }}$ corresponding to increasing erythroid differentiation. GPA $^{\text {low }}$ population was only present at $0 \mathrm{~h}$. Treatment of EpoCD34/HSPCs with $\mathrm{TNF} \alpha$ or

C2-ceramide for $96 \mathrm{~h}$ reduced the formation of the GPA ${ }^{\text {bright }}$ population from 23 to $15 \%$, while dihydroceramide had no effect (Fig. 1g). Furthermore, TNF $\alpha, \mathrm{C} 2$-ceramide, and 
Fig. 1 a Mass spectrometry analysis of C16-, C16:1-, C18-, C18:1-, C20-, C22-, C24-, C24:1-, C26-, and C26:1-ceramide in EpoCD34/ HSPCs treated with TNF $\alpha$ and GW4869 for $24 \mathrm{~h}$. Ceramide species were quantified by ESI-MS/MS and results were reported to the number of cells. EpoCD34/HSPCs (Epo: $2 \mathrm{U} / \mathrm{mL}$ ) were untreated (U) or treated as indicated with TNF $\alpha$, GW4869, desipramine, C2 ceramide, bSMase, dihydroceramide, sphingosine-1-phosphate (S1P), or PF-543 or transfected with si-SMPD3. b Benzidine staining assays with sphingomyelinase inhibitors at $96 \mathrm{~h}$. $\mathbf{c}$ Benzidine staining assays with si-SMPD3 at $96 \mathrm{~h}$. d Comparison of TNF $\alpha$ effect on benzidine staining assays at $96 \mathrm{~h}$ with ceramides and its derivatives. e Benzidine staining assays with S1P and PF-543 at $96 \mathrm{~h}$. f Real-Time PCR and Western blot analysis of SphK1 expression at $24 \mathrm{~h}$. g Flow cytometry analysis of GPA expression. GPA-expressing populations were identified as low (lo), medium (me), and bright (br). Mean of percentage \pm $\mathrm{SD}$ of GPA ${ }^{\text {bright }}$ cells is indicated in each quadrant. $\mathbf{h}$ Real-time PCR analysis of globins and EpoR expression at $96 \mathrm{~h}$. i Western blot analysis of globin's expression at $96 \mathrm{~h}$. Bars represent the mean $\pm \mathrm{SD}$ of four independent experiments except for $\mathbf{f}$ with three independent experiments. One-way ANOVA with repeated measures followed by Dunnett's post hoc test show the statistical significance ${ }^{*}, \$ P<0.05$, ***, ${ }^{\$} P<0.01$ (*: treatment vs. untreated; $\$$ : treatment vs. TNF $\alpha$ ). A Student's $t$ test was performed for $\mathbf{f}$. $\beta$-Actin expression was used in Western blot analyses as an internal control and was revealed each time on the same membrane than one or several proteins of interest

bSMase strongly reduced both mRNA and protein expression of erythroid markers (globins and EpoR) (Fig. 1h, j). Similarly, C6-ceramide decreased globin expression (Supplementary Figure S3b). In contrast, S1P and GW4869 prevented this effect while desipramine did not. To further investigate the impact of the $\mathrm{TNF} \alpha /$ ceramide pathway on erythropoiesis, May-Grünwald-Giemsa (MGG) staining assays were performed after 9 days. Results showed abundant orthochromatophilic erythroblasts, enucleation pictures, and reticulocytes in EpoCD34/HSPCs at day 9 compared to day 0 (Fig. 2a). TNF $\alpha, \mathrm{C} 2$-ceramide, and bSMase decreased the total number of erythroid cells by 55 , 40 , and $60 \%$, respectively. Orthochromatophilic erythroblasts represented only 7 and $11 \%$ after TNF $\alpha$ and C2ceramide treatment, respectively. bSMase completely abolished their production. Moreover, no reticulocyte or enucleation was identified under those conditions. GW4869 prevented the effect of TNF $\alpha$ with the restoration of $75 \%$ of erythroid cells at all maturation stages. Conversely, desipramine reinforced the effect of TNF $\alpha$ (Fig. 2b).

Our results support a fundamental role for the nSMase/ ceramide pathway in $\mathrm{TNF} \alpha$-mediated impairment of erythropoiesis. Furthermore, TNF $\alpha$ perturbates the sphingolipid rheostat by inhibiting SphK1 expression leading to ceramide accumulation, exacerbating erythropoiesis inhibition.

\section{The TNFa/nSMase/ceramide pathway converts erythropoiesis to myelopoiesis}

Considering that TNF $\alpha$ and ceramides reduced erythroid differentiation in EpoCD34/HSPCs without inducing cell death (Supplementary Figure S3c and S4), we assessed the fate of the remaining non-erythroid cell population. Following TNF $\alpha$, C2-ceramide, and bSMase treatments, MGG staining revealed different stages of myeloid maturation including polymorphonuclear neutrophils (Fig. 2a, b). Colony formation assays with EpoCD34/HSPCs confirmed the distribution of cellular differentiation. Assays displayed $79 \%$ of burstforming unit-erythroid/colony-forming unit-erythroid (BFUE/CFU-E) colonies, while the remaining colonies were represented by CFU-granulocyte-erythrocyte-monocytemegakaryocyte (GEMM), CFU-GM, CFU-G, and CFU-M after 14 days (Fig. 2c, d). Following TNF $\alpha, \mathrm{C} 2$-ceramide, and bSMase treatments, myeloid colonies CFU-G/CFU-M increased by 5-, 3.5- and 8-fold, respectively, concomitant with a decrease of BFU-E/CFU-E colonies reaching 41, 51 and $22.5 \%$, respectively (Fig. 2d). Moreover, S1P treatment prevented the decrease of BFU-E formation. Importantly, GW4869 efficiently prevented the effect of TNFo by restoring the score of BFU-E/CFU-E to 78\%, whereas desipramine reduced BFU-E/CFU-E formation concurrently to an enhancement of myeloid subpopulations (Fig. 2d and Supplementary Table S2). TNF $\alpha$ and ceramides significantly increased CD11b expression from $96 \mathrm{~h}$ and GW4869 prevented the induction (Fig. 2e and Supplementary Figure S3d). Furthermore, nSMase inhibition by GW4869 and knock down by si-SMPD3 also prevented TNFo-induced neutrophil differentiation as shown by nitro blue tetrazolium (NBT) staining and the downregulation of matrix metalloproteinase (MMP)-9 gene (Fig. 2f, g and Supplementary Figure S5). Our

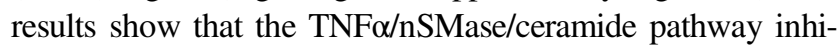
bits erythropoiesis concomitantly with granulomonocytic differentiation.

\section{Myeloid differentiation and inhibition of erythropoiesis correlate with the modulation of related TFs and miRs}

We then considered the conversion of erythroid to myeloid differentiation in EpoCD34/HSPCs treated by TNF $\alpha$ and ceramides. The effect of the TNF $\alpha /$ ceramide pathway was analyzed via the expression of key regulators of myelopoiesis and erythropoiesis, including TFs PU.1, GATA-1, and GATA-2, as well as related miRs at different time points (Fig. 2h, i and Supplementary Figure S6). TF expression was not affected after $24 \mathrm{~h}$ by TNF $\alpha$ (Supplementary Figure S6). At $96 \mathrm{~h}$, expression of the erythropoiesis repressors GATA-2 and PU.1 were induced in TNF $\alpha$ treated cells in a nSMase-dependent manner as shown in GW4869-co-treated cells. PU.1 was also up-regulated by C2-ceramide and bSMase, in correlation with both the inhibitory effect on erythropoiesis and the induction of myelopoiesis. GATA-2 expression was induced by bSMase treatment, while $\mathrm{C} 2$-ceramide had no effect. Even though 


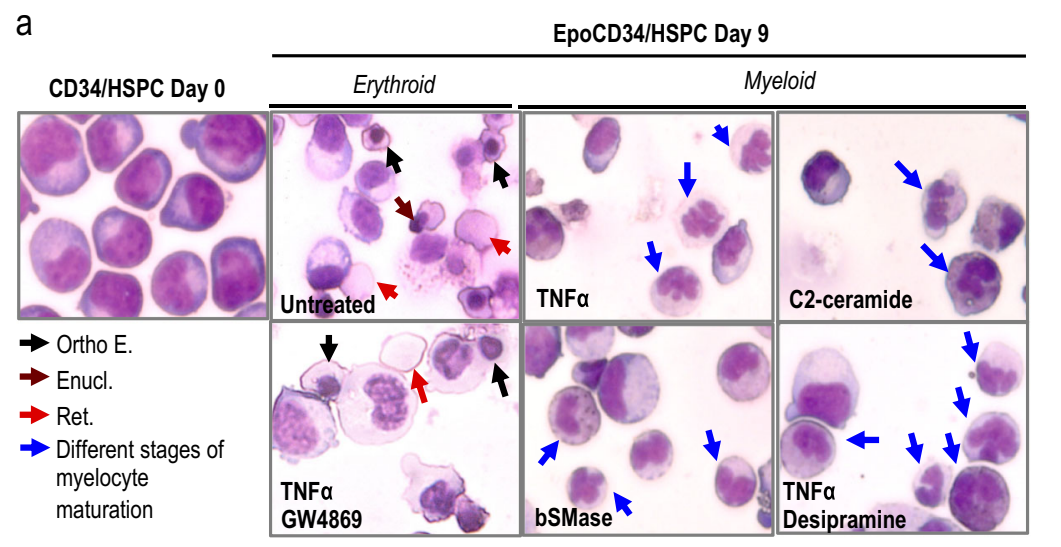

C
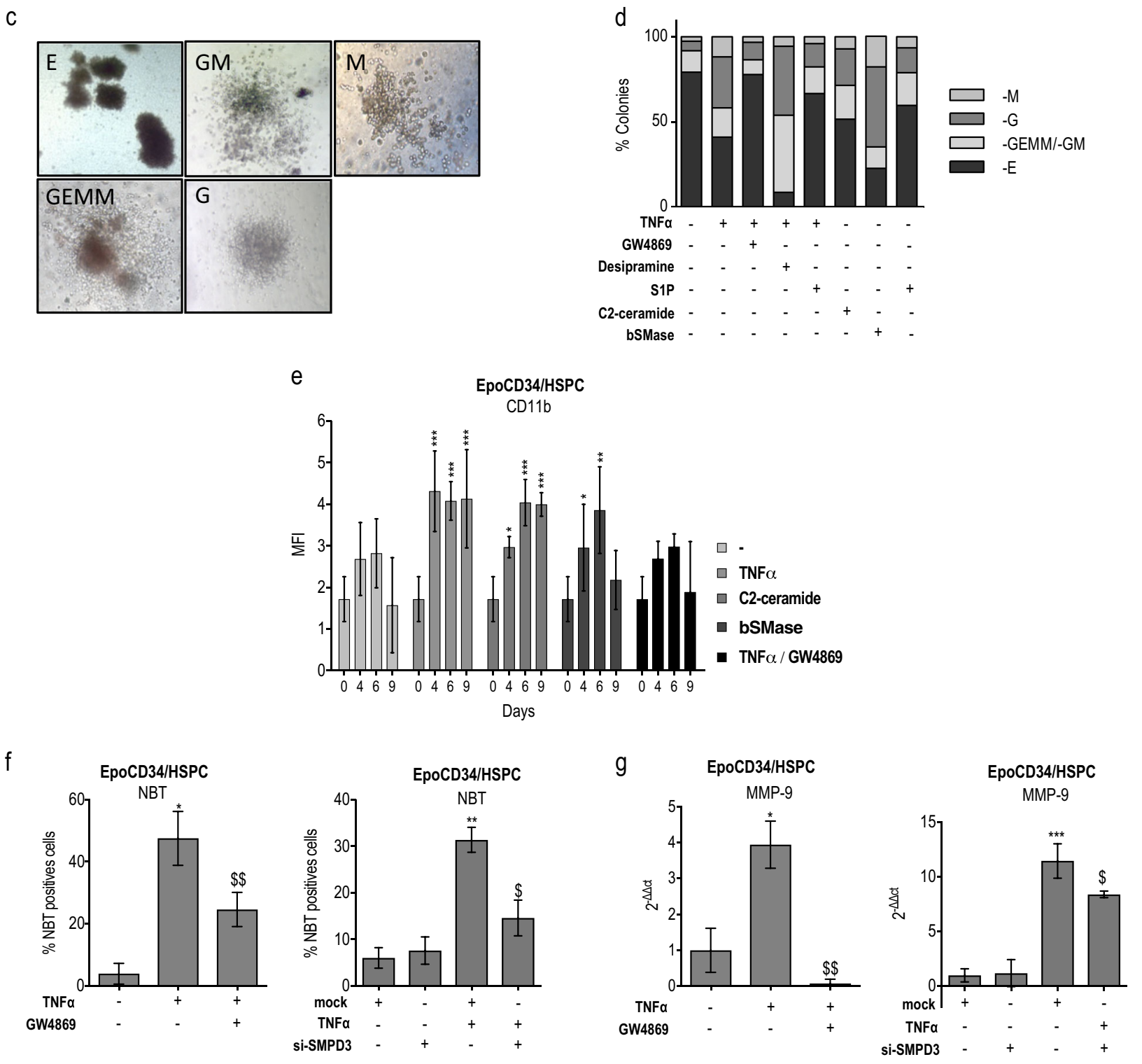

cells were committed towards the myeloid lineage and erythroid-specific genes were downregulated, GATA-1 remained expressed after $96 \mathrm{~h}$ of $\mathrm{TNF} \alpha$ treatment, in b

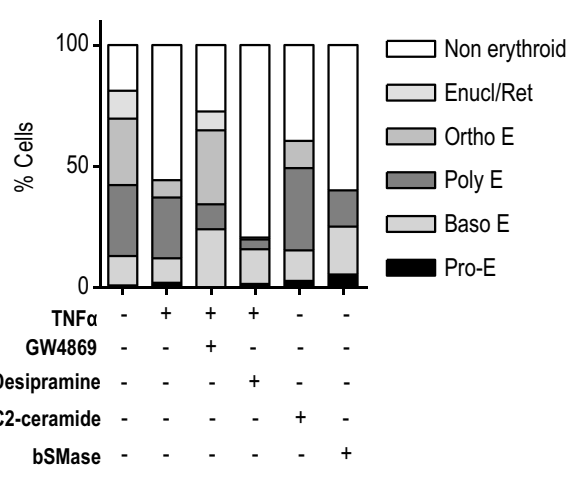


h

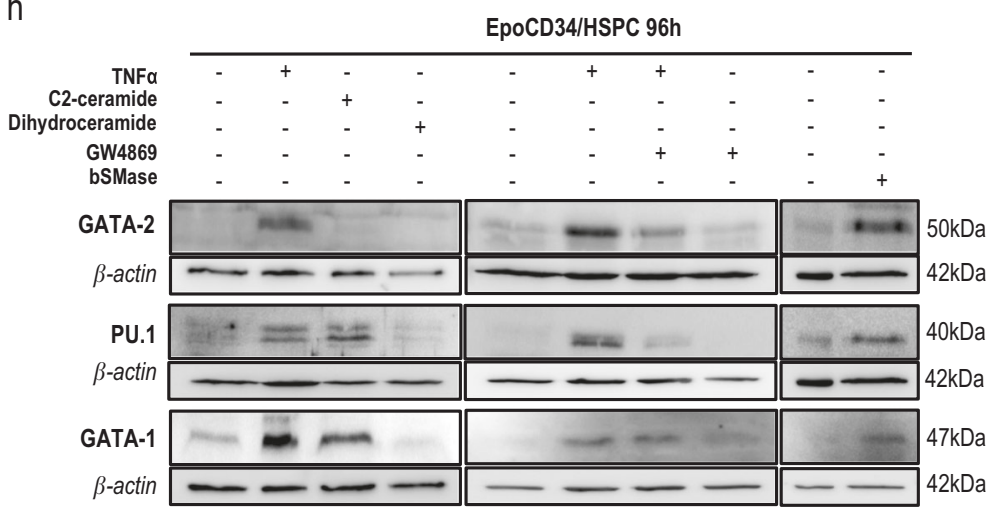

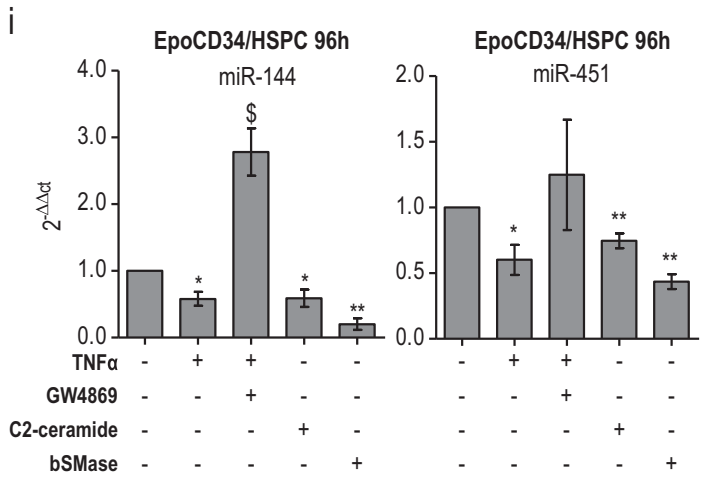

Fig. 2 Impact of $\mathrm{TNF} \alpha / \mathrm{SMase} /$ ceramide pathway on EpoCD34/ HSPCs differentiation. a May-Grünwald-Giemsa staining of CD34/ HSPCs at day 0 and EpoCD34/HSPCs at day 9 of treatment observed with a $\times 40$ objective. Each picture is representative of three independent experiments. Enucl/Ret: enucleation/reticulocyte; E: erythroblast; Ortho: orthochromatophilic. b Representation of the different cell types distribution on MGG-stained slides. c Microscope observation $(\times 20)$ of distinctive morphological aspects of hematopoietic colonies obtained after EpoCD34/HSPCs treatments at day 14. E: erythroid; GM: granulocyte-monocyte; GEMM: granulocyte-erythrocyte-monocyte-megakaryocyte; G: granulocyte; M: monocyte/ macrophage. d Representation of the different colonies distribution in colony formation assays of EpoCD34/HSPCs treatments at day 14. e Flow cytometry analysis of CD11b expression in EpoCD34/HSPCs

In agreement with the impairment of erythropoiesis and the myeloid commitment, hematopoiesis-related miRs were also modulated. $\mathrm{TNF} \alpha, \mathrm{C} 2$-ceramide, and bSMase inhibited the expression of the positive regulators of erythropoiesis miR-144 and miR-451, which are regulated by GATA-1. Accordingly, GW4869 prevented $\mathrm{TNF} \alpha$-mediated inhibition of these miRs. TNF $\alpha$ induced the expression of the myeloid-specific miR-146a, miR-155, and miR-223, controlled by PU.1. Likewise, GW4869 prevented the upregulation of these miRs. However, C2-ceramide and bSMase did not affect miR146a and miR-155, but induced the granulocytic miR-223 (Fig. 2i). Results suggested that the TNF $\alpha / n S M a s e / c e r-$ amide pathway modulates key regulators of both myeloid differentiation and erythropoiesis. from day 0 to 9 of culture. f NBT staining with GW4869 and siSMPD3 at $96 \mathrm{~h}$. g Real-time PCR analysis of MMP-9 expression with GW4869 and si-SMPD3 at $96 \mathrm{~h}$. h Western blot analysis of GATA-1, GATA-2, and PU.1 in EpoCD34/HSPCs after $96 \mathrm{~h}$ of treatment. $\beta$ Actin expression was used in Western blot analyses as an internal control and was revealed each time on the same membrane than one or several proteins of interest. i Real-time PCR analysis of miR-144, $-451,-155,-146 \mathrm{a}$, and -223 expression levels in EpoCD34/HSPCs. Bars represent the mean \pm SD of five independent experiments. Oneway ANOVA with repeated measures followed by Dunnett's post hoc test or two-way ANOVA followed by Holm-Sidak's post hoc test show the statistical significance $*, \$ P<0.05, * * P<0.01$, $* * * P<0.001$ $(*$ : treatment vs. untreated, $\$$ : treatment vs. TNF $\alpha$ )

\section{The TNFa/nSMase/ceramide pathway impairs autophagy}

Autophagy is critical for erythropoiesis and was reported as inversely correlated to neutrophil differentiation [17]. Thus, we assessed the capacity of the TNF $\alpha / n S M a s e / c e r-$ amide pathway to impair autophagy.

The ability of Epo to induce an autophagic flux was first evaluated by analyzing LC 3 conversion at $0,24,72,96$, and $120 \mathrm{~h}$ in the presence or absence of bafilomycin A1 (BafA1) by Western blot (Supplementary Figure S7) where the accumulation of LC3-II increased with time. To assess the effect of TNF $\alpha$ and GW4869 on Epo-mediated autophagy in CD34/HSPCs, we performed Western blot analysis of LC3 conversion. Results showed that in the presence 
a

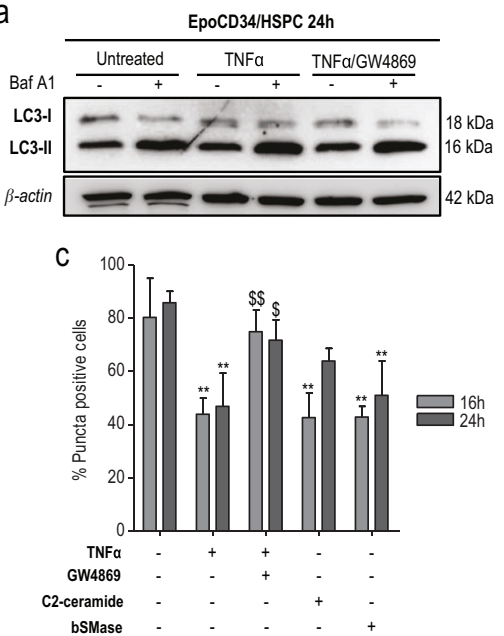

b

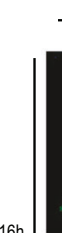

$16 \mathrm{~h}$

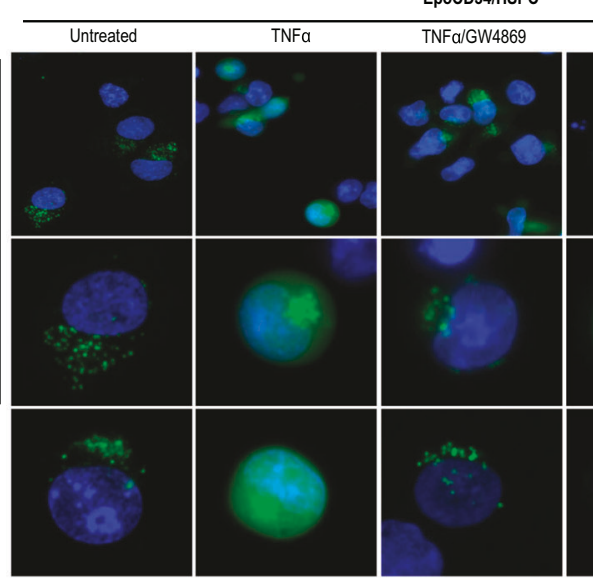

CD34/HSPC Oh GFP-LC3

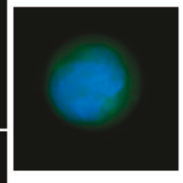

GFP
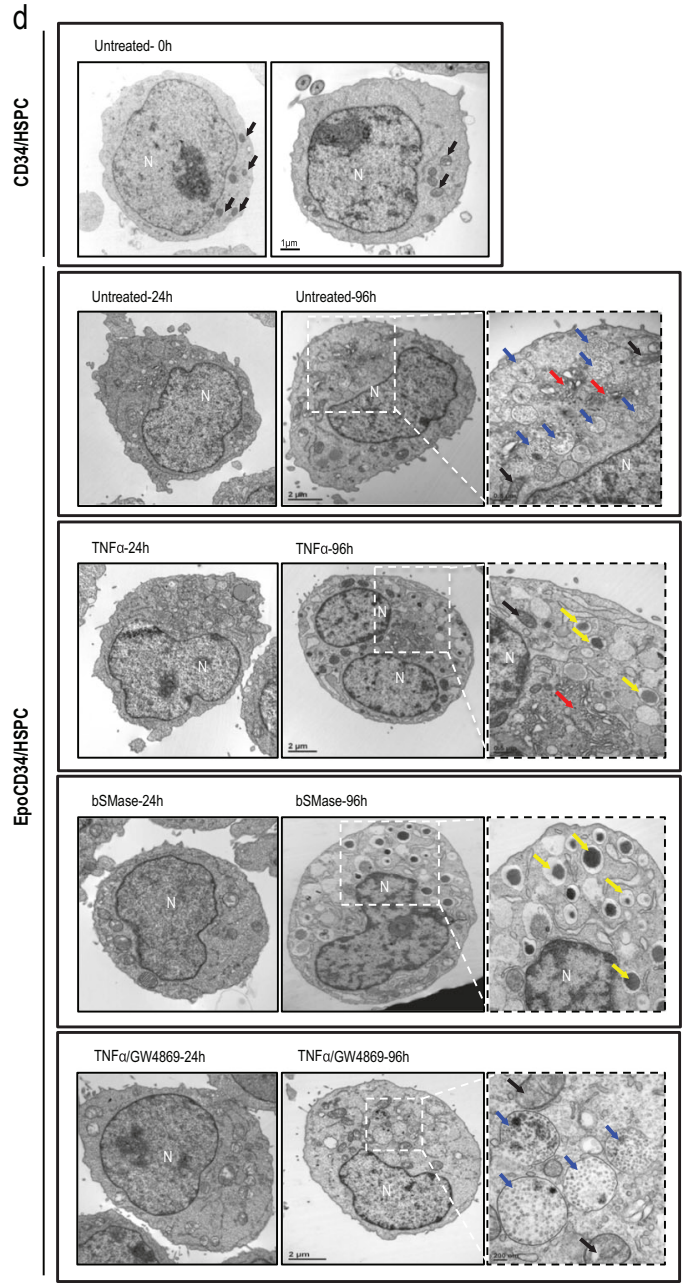

of BafA1, LC3-II accumulated in all conditions including in untreated and TNF $\alpha$-treated cells at $24 \mathrm{~h}$ (Fig. 3a). Therefore, we assessed the effect of TNF $\alpha, \mathrm{C} 2$-ceramide, bSMase, and GW4869 on autophagy by transfecting CD34/HSPCs with a GFP-LC3 expression construct. Fluorescence microscopy allowed to quantify cells with
GFP-LC3 punctae at 16 and $24 \mathrm{~h}$ visible in $80 \%$ of transfected EpoCD34/HSPCs vs. 3\% in CD34/HSPCs (Fig. 3b, c). $\mathrm{TNF} \alpha, \mathrm{C} 2$-ceramide, and bSMase significantly reduced the percentage of cells displaying GFP-LC3 punctae by $50 \%$. The inhibition of nSMase by GW4869 prevented the effect of TNF $\alpha$ as shown by the restoration of punctae 
Fig. 3 Impact of $\mathrm{TNF} \alpha / \mathrm{SMase} / \mathrm{ceramide}$ pathway on EpoCD34/ HSPCs autophagy. a Western blot analysis of LC3 conversion in EpoCD34/HSPCs treated as indicated for $24 \mathrm{~h}$. BafA1: bafilomycin A1. $\beta$-Actin expression was used in Western blot analyses as an internal control. b Fluorescence microscopy observations of Epo-free or EpoCD34/HSPCs after nucleofection with pSelect-GFP-LC3 plasmid or with GFP control plasmid and treatments as indicated for 0,16 , and $24 \mathrm{~h}$. Nuclei were stained with 4',6-diamidino-2-phenylindole (DAPI). Two lower rows show magnified cells representative of the transfected population $(\times 40)$; in row 1 , cells are shown in a larger field at $24 \mathrm{~h}(\times 20)$. c Quantification of transfected viable cells that display punctae pattern without diffuse signal. Bars represent the mean \pm SD of three independent experiments. Two-way ANOVA followed by Holm-Sidak's post hoc test show the statistical significance $*, \$ P<$ $0.05,{ }^{*}, \$ \$ P<0.01(*$ : vs. untreated, $\$$ : vs. TNF $\alpha$ ). d TEM pictures of untreated CD34/HSPCs at $0 \mathrm{~h}$ and of untreated or treated as indicated EpoCD34/HSPCs at $24 \mathrm{~h}$ and $96 \mathrm{~h}$. N: nucleus; blue arrows: autophagosome/autolysosome, black arrows: mitochondria, red arrows: Golgi, yellow arrows: dense-core early azurophilic granules and secretion vesicles

formation. These results suggested that $\mathrm{TNF} \alpha$ and ceramides inhibit autophagy in EpoCD34/HSPCs. To further confirm these results, we performed observations by transmission electron microscopy (TEM) (Fig. 3d). The typical phenotype of immature HSPCs could be observed at $0 \mathrm{~h}$ with a large nucleus and early structures of autophagy (phagophores), which remained visible at $24 \mathrm{~h}$ in EpoCD34/HSPCs whatever the treatment used. At $96 \mathrm{~h}$, we observed abundant autophagosome/autolysosome accumulation in EpoCD34/HSPCs confirming activation of autophagy. Following TNF $\alpha$ and bSMase treatments, the absence of autophagosome/autolysosome accumulation suggested an impairment of autophagy. Moreover, densecore early azurophilic granules in the Golgi region as well as the nuclear lobulation suggested a promyelocytic differentiation. Interestingly, GW4869 prevented the inhibition of autophagosome formation. We document here that the $\mathrm{TNF} \alpha /$ ceramide pathway inhibits autophagy in EpoCD34/HSPCs concomitantly with inhibition of erythropoiesis.

\section{The TNFa/nSMase/ceramide pathway impairs autophagy via mTOR activation}

We then investigated the mechanistic aspects of the inhi-

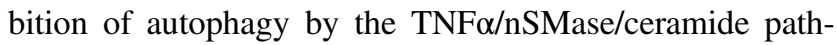
way. At $24 \mathrm{~h}$, we observed that TNF $\alpha$ increased phosphorylation of PI3K and mTOR. The PI3K/AKT/ mTOR/ULK1 ${ }^{\mathrm{S} 758}$ pathway was fully activated after $96 \mathrm{~h}$, leading to inhibition of $\mathrm{Atg} 13^{\mathrm{S} 355}$ phosphorylation, thus affecting autophagy (Fig. 4a, Supplementary Figure S8). Treatments with bSMase also induced phosphorylation of $\mathrm{mTOR}^{\mathrm{S} 2448}$ and $\mathrm{ULK} 1^{\mathrm{S} 758}$ and subsequent inhibition of Atg $13^{\mathrm{S} 355}$ phosphorylation, but this effect was independent of PI3K/AKT pathway activation. Inhibition of nSMase in
TNF $\alpha$-treated cells through pharmacological or genetic inhibition with GW4869 or si-SMPD3 prevented the effect of $\mathrm{TNF} \alpha$ on $\mathrm{mTOR}^{\mathrm{S} 2448} / \mathrm{ULK}^{\mathrm{S} 758}$ phosphorylation, supporting the implication of the nSMase pathway in autophagy impairment (Fig. 4a).

Analysis of autophagy-related proteins showed that Beclin-1 expression and Atg5-Atg12 complex formation was induced in Epo-treated CD34/HSPCs at $24 \mathrm{~h}$ compared to $0 \mathrm{~h}$, while Rubicon expression was abolished. At $24 \mathrm{~h}$, $\mathrm{TNF} \alpha$ and bSMase induced an inhibition of Beclin-1 and Atg5-Atg12, which did not persist at $96 \mathrm{~h}$. Atg7 was not affected. Interestingly, p62/SQSTM1 protein remained undetectable in untreated EpoCD34/HSPCs at $96 \mathrm{~h}$, while it accumulated in TNF $\alpha$ - and bSMase-treated cells witnessing an inhibition of autophagy. Similarly, Rubicon expression was strongly induced at $96 \mathrm{~h}$ by TNF $\alpha$ treatment compared to untreated cells in agreement with autophagy inhibition (Fig. 4b). Activation of the mTOR pathway transduced the repressive potential of TNF $\alpha$ and ceramides on Epo-mediated physiological autophagy.

\section{Autophagy is required for TNFa/ceramide-mediated modulation of differentiation}

We then assessed the requirement of autophagy in the effect of the $\mathrm{TNF} \alpha / \mathrm{nSMase} /$ ceramide pathway on CD34/HSPC differentiation. The efficiency of rapamycin as an mTOR inhibitor and autophagy inducer was first verified in our model under different conditions by GFP-LC3 transfection and fluorescent microscopy (Fig. 5a). We assessed the ability of the mTOR inhibitor rapamycin to restore autophagy in TNF $\alpha$-treated cells by performing GFP-LC3 transfections in EpoCD34/HSPCs. Numeration of cells displaying punctae at $16 \mathrm{~h}$ by fluorescence microscopy showed that rapamycin restored punctae formation in $\mathrm{TNF} \alpha$-treated cells, verifying simultaneously the ability of rapamycin to induce autophagy in our model. Rapamycin had no significant effect in Epo-treated cells that already presented autophagic features.

We then analyzed the ability of rapamycin-induced autophagy to prevent inhibition of erythropoiesis in EpoCD34/HSPCs treated by TNF $\alpha$ or bSMase for $96 \mathrm{~h}$. Rapamycin was able to restore erythroid differentiation in TNF $\alpha$ - and bSMase-treated cells as shown by CFU-E/ BFU-E formation and the partial restoration of hemoglobinproducing cells (Fig. 5b, c). Results showed that rapamycininduced autophagy in TNF $\alpha$-treated cells correlated with induced erythroid differentiation. Concomitantly, the myeloid colonies and $\mathrm{CD} 11 \mathrm{~b}$ expression were reduced (Fig. 5b, d). Conversely, inhibition of the late stage of autophagy by BafA1 strongly decreased the amount of Epo-induced CFU-E/BFU-E formation as well as the percentage of hemoglobin-producing cells while favoring 
Fig. 4 Western blot analysis of autophagyrelated proteins expression in EpoCD34/ HSPCs. a PI3K/AKT/mTOR/ULK1/Atg13 pathway of untreated or treated cells as indicated or transfected with si-SMPD3 at $96 \mathrm{~h}$. b Beclin-1, Atg5-12, Atg7, p62/SQSTM1, and Rubicon in untreated CD34/HSPCs at $0 \mathrm{~h}$ and in EpoCD34/HSPCs untreated or treated as indicated at 24 and $96 \mathrm{~h}$. $\beta$-Actin expression was used in Western blot analysis as an internal control and was revealed each time on the same membrane than one or several proteins of interest a

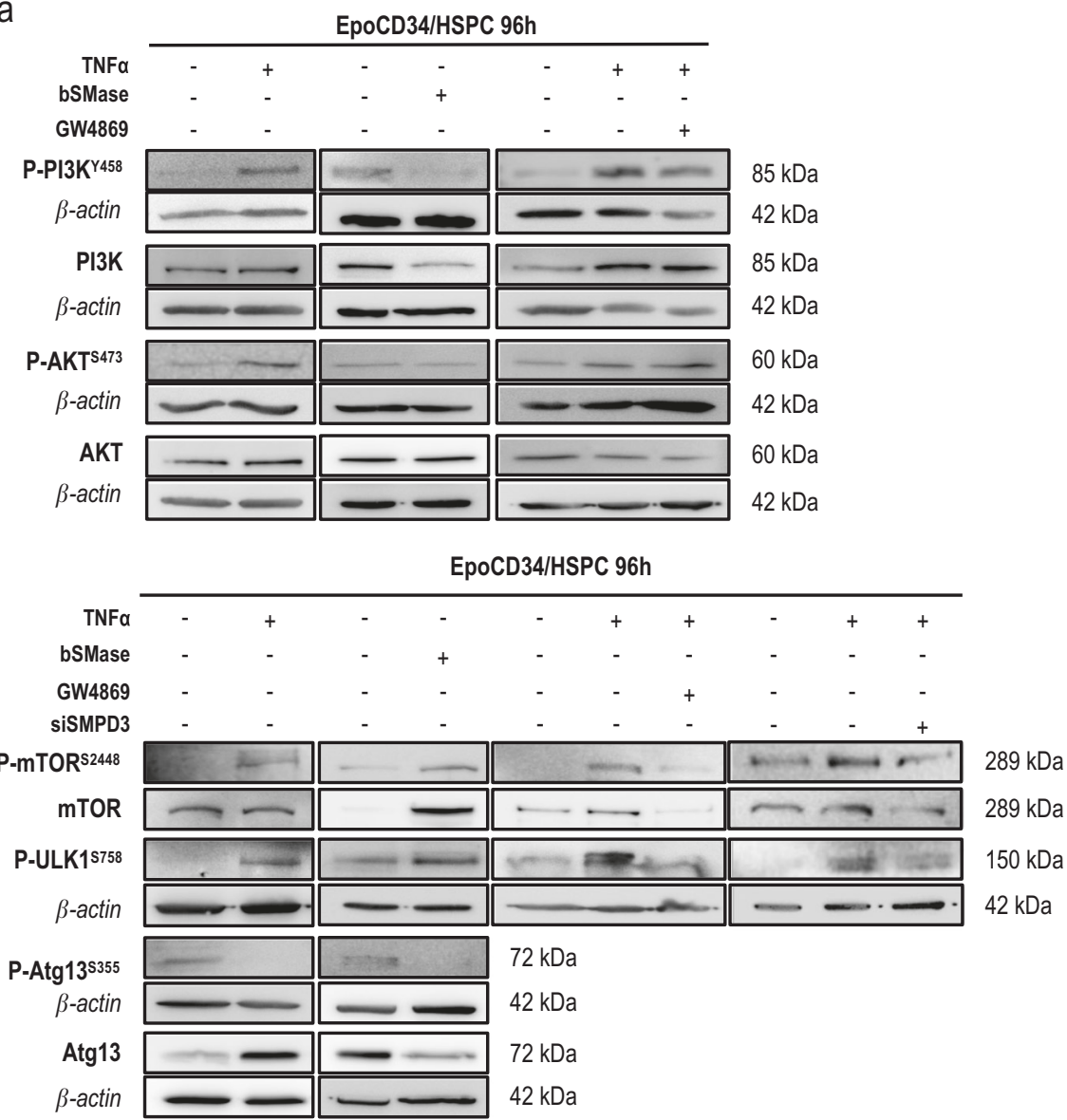

b

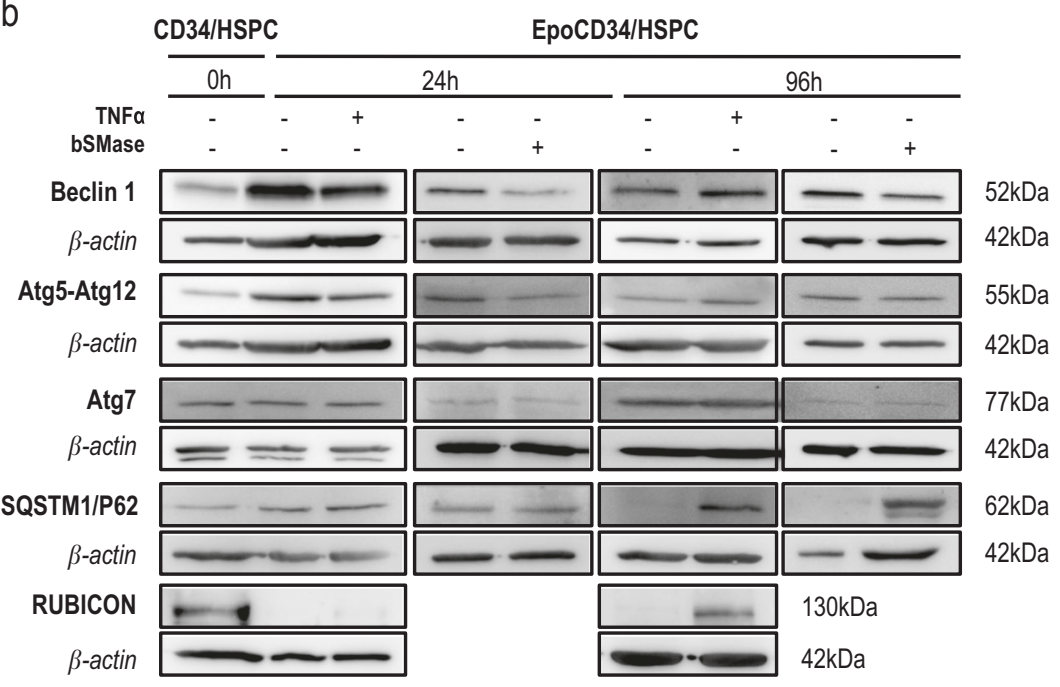

myeloid colonies (Fig. 5b-d). On the other hand, we set up CD34/HSPC transfection with siRNA against Atg5 (si-Atg5) and verified its efficiency on Atg5 expression (mRNA and protein) as well as its ability to inhibit autophagy by LC3 conversion analysis (Supplementary Figure S2). Interestingly, knock down of the earlystep autophagy Atg5 gene with si-Atg5 affected erythroid differentiation as shown by reduced benzidine staining and decreased $\alpha$-globin mRNA expression (Fig. 5c). EpoCD34/ HSPCs treated with BafA1 displayed an increase in granulocytic intracytoplasmic deposits of reduced formazan (NBT staining), whereas this marker and MMP-9 mRNA expression were not significantly affected in si-Atg5transfected cells (Fig. 5e). Co-treatment of EpoCD34/ 
a

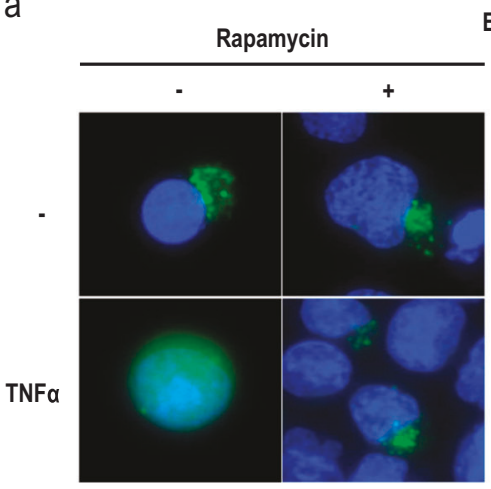

C
EpoCD34/HSPC 16h

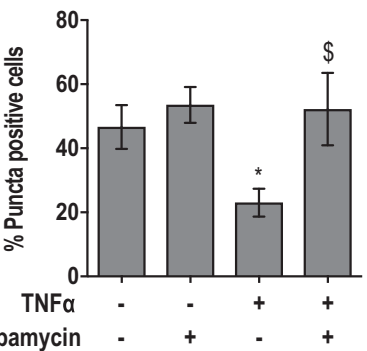

b

EpoCD34/HSPC Day 14

CFA

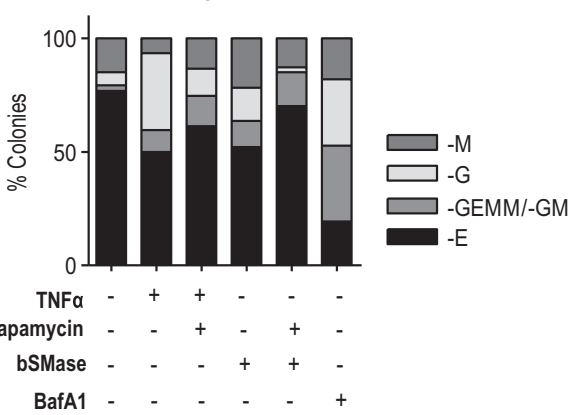

EpoCD34/HSPC 96h
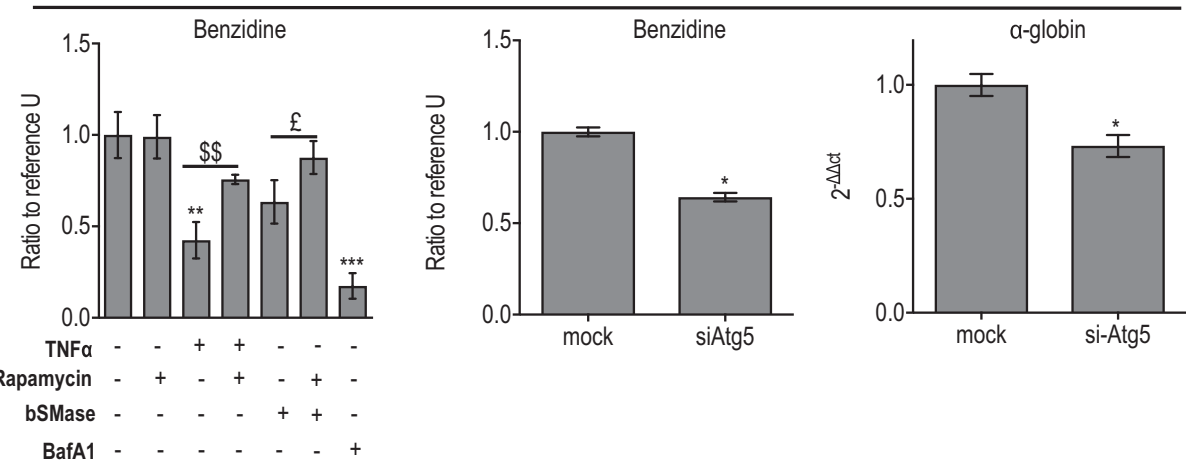

d
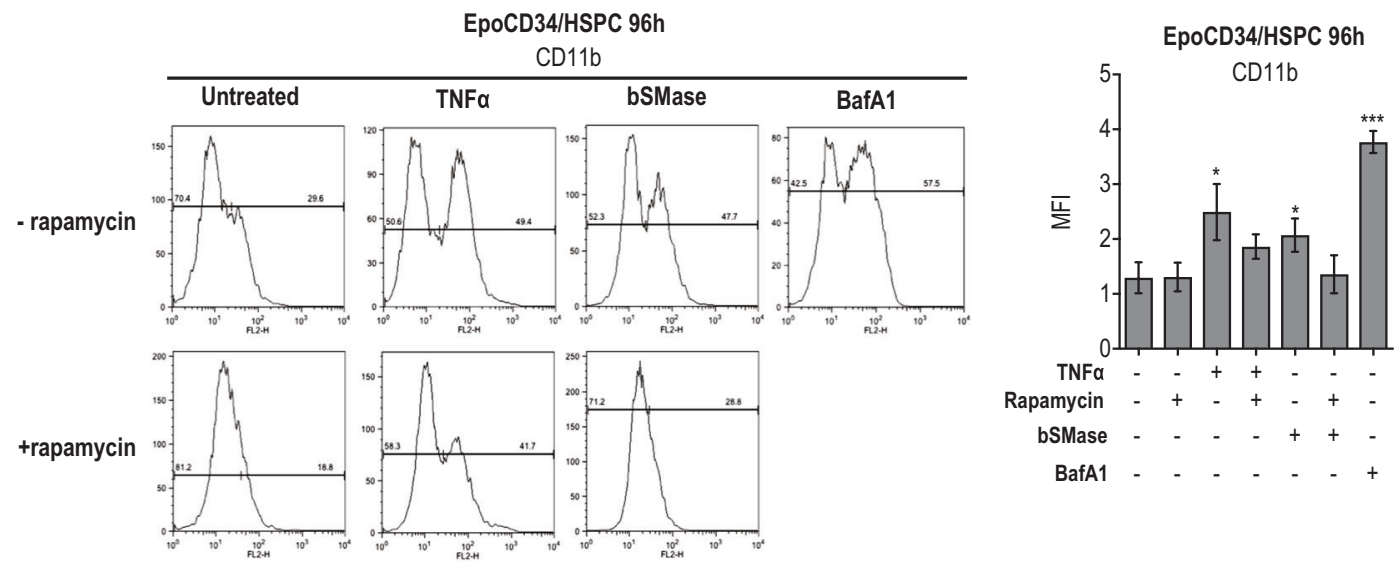

e

EpoCD34/HSPC 96h
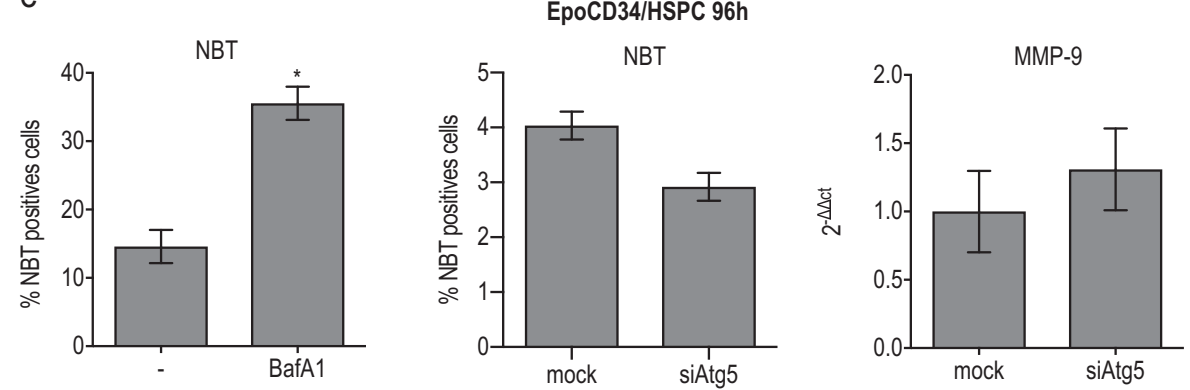

HSPCs with TNF $\alpha$ or bSMase and rapamycin had no effect on cell viability and BafA1 reduced cell viability by $15 \%$ at 96 h (Supplementary Figure S4).
These results underlined that inhibition of erythropoiesis resulted from $\mathrm{TNF} \alpha /$ ceramide-induced autophagy impairment. Besides, our data suggest that granulocyte 
Fig. 5 Impact of autophagy on erythroid vs. myeloid commitment. EpoCD34/HSPCs were treated with bafilomycin A1 (BafA1) and with rapamycin in the presence or absence of TNF $\alpha$ or bSMase during $96 \mathrm{~h}$. a Fluorescence microscopy observation of EpoCD34/HSPCs after nucleofection with pSelect-GFP-LC3 plasmid and treatments with $\mathrm{TNF} \alpha$ and/or rapamycin for $16 \mathrm{~h}$. Nuclei were stained with $4^{\prime}, 6$-diamidino-2-phenylindole (DAPI). Cells were observed with $\times 40$ objective. Transfected viable cells that display punctae pattern without diffuse signal were quantified. Bars represent the mean $\pm \mathrm{SD}$ of three independent experiments. Two-way ANOVA followed by HolmSidak's post hoc test show the statistical significance ${ }^{*}, \$<0.05$ (*: vs. untreated, \$: vs. TNF $\alpha$ ). b Colony formation assays at day 14 . c Benzidine staining and real-time PCR analysis of $\alpha$-globin mRNA expression in the presence of autophagy modulators at $96 \mathrm{~h}$. d Flow cytometry analysis of CD11b expression in EpoCD34/HSPCs at $96 \mathrm{~h}$. e NBT staining and real-time PCR analysis of MMP-9 mRNA expression with bafilomycin A1 (BafA1) and si-Atg5 at $96 \mathrm{~h}$. Bars represent the mean $\pm \mathrm{SD}$ of three or five independent experiments. One-way ANOVA with repeated measures followed by Dunnett's post

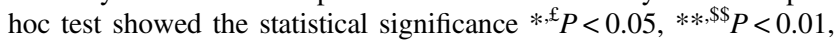
$* * * P<0.001$ (*: vs. untreated, \$: vs. TNF $\alpha, £$ : vs. bSMase)

differentiation depends on late autophagy inhibition since inhibition of the early-step regulator Atg5 had no effect on granulocyte differentiation.

\section{Discussion}

The molecular mechanisms describing how ceramides regulate $\mathrm{TNF} \alpha$-mediated conversion of erythropoiesis to myelopoiesis in EpoCD34/HSPCs through inhibition of autophagy and modulation of specific TFs/miRs are summarized Fig. 6.

We show that exogenous $\mathrm{C} 2$ - and C6-ceramide and bSMase-induced endogenous ceramides prevented the expression of erythroid markers, erythropoiesis features, and miR-144/451 expression in EpoCD34/HSPCs similarly to TNFo. Moreover, nSMase inhibitor GW4869 and siRNA targeting nSMase showed that the TNF $\alpha / n S M a s e$ pathway regulates the inhibition of erythropoiesis. Ceramides were previously described to inhibit erythropoiesis, induce apoptosis, and reduce CFU-E formation from peripheral blood mononuclear cells [32].

Importantly, whatever the treatment used here, cell viability remained unaffected, excluding involvement of apoptosis in erythropoiesis inhibition.

Besides ceramides, other derivatives such as S1P are involved in cell differentiation and autophagy [30]. Blockade of S1P formation by inhibiting SphK1 prevented erythroid differentiation and enhanced the effect of $\mathrm{TNF} \alpha$, supporting a positive role for S1P in erythropoiesis. Interestingly, $\mathrm{TNF} \alpha$ also inhibited the expression of SphK1, which is regulated by GATA-1 [33]. Similarly, treatments with S1P prevented the inhibition of erythropoiesis by $\mathrm{TNF} \alpha$ in agreement with its antagonistic effect on ceramides [31]. On the other hand, ceramidases are unable to generate sphingosine upon hydrolysis of C2-ceramide, so that this intermediate metabolite is likely not involved in erythropoiesis inhibition [34]. Altogether, these results hint at ceramides as the main bioactive sphingolipids involved in $\mathrm{TNF} \alpha$-mediated inhibition of erythropoiesis.

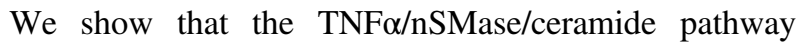
leads both to the inhibition of Epo-activated erythropoiesis and the activation of myeloid differentiation. In agreement with our results, TNF $\alpha$ and Vitamin D3, an inducer of granulocyte differentiation, were reported to activate ceramide metabolism in HL60 cells [35, 36]. Remarkably, granulopoiesis was enhanced by the PI3K/AKT/mTOR cascade and by overexpression of AKT in murine CD34/ HSPCs, whereas an inhibition of AKT led to the absence of differentiation [37-39]. Here, TNF $\alpha$-mediated activation of PI3K/AKT/mTOR and p62/SQSTM1 accumulation correlated with granulocytic differentiation [40].

Results show that $\mathrm{TNF} \alpha$ and ceramides induce granulopoiesis despite the presence of Epo, suggesting a role for the $\mathrm{TNF} \alpha / \mathrm{nSMase} /$ ceramide pathway in cell fate determination. According to their interaction and cooperation modalities, TFs GATA-1, GATA-2, and PU.1 are essential parameters for hematopoietic cell commitment [41-43]. Modulation of these TFs is correlated with $\mathrm{TNF} \alpha /$ ceramide-mediated impairment of erythropoiesis and activation of myelopoiesis. Despite the active role of GATA-1 in erythroid gene transcription, our results also showed increased GATA-1 expression after $\mathrm{TNF} \alpha, \mathrm{C} 2$-ceramide, and bSMase treatments in agreement with reports describing that GATA-1 overexpression inhibited erythropoiesis in mouse erythroblasts culminating in lethal anemia [44]. Besides, our results correlate with an inhibition of GATA-1 activity. Indeed, GATA-1 target genes, including erythroid-specific genes, $m i R-144 / 451$ and $\operatorname{SphK1}$, are downregulated. Furthermore, impairment of GATA-1 activity can be explained by both GATA-2 and PU.1 overexpression. Indeed, active GATA-1 inhibits GATA-2 transcription, while overexpression of GATA-2 inhibits erythropoiesis [8, 45]. Moreover, GATA2 activates GATA-1 transcription. As a result, GATA-2 overexpression in $\mathrm{TNF} \alpha$ - and ceramide-treated cells leads to GATA-1 overexpression in agreement with both GATA-1 inactivation and inhibition of erythropoiesis. Besides, loss of GATA-1 activity correlates with induction of the myeloid TF PU.1, which inhibits GATA-1 through physical interaction $[10,46]$. We previously reported that $\mathrm{TNF} \alpha$ promoted the GATA-1/PU.1 inhibitory complex in EpoCD34/ HSPCs [2].

Obviously, increased PU.1 levels not only coincided with erythropoiesis inhibition but also triggered myelopoiesis with CD11b, miR-223, miR-146a, and miR-155 upregulation [47]. TNF $\alpha$ and ceramides induced miR-223, while 


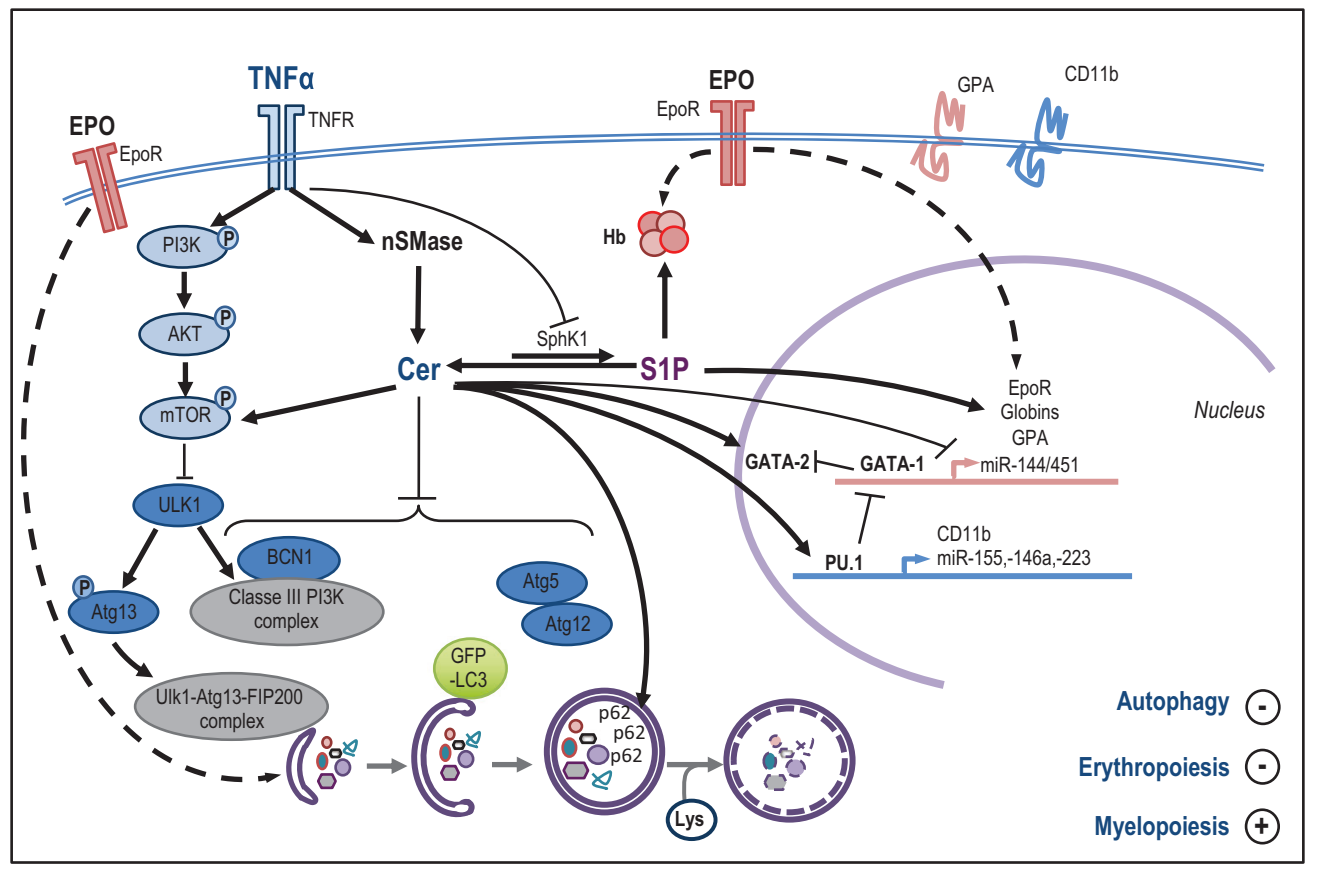

Fig. 6 Erythropoietin (Epo) binds to the Epo receptor (EpoR) to activate erythroid-specific gene transcription (EpoR, globins, glycophorin A (GPA), and miR-144/451) by GATA-1, resulting in hemoglobin $(\mathrm{Hb})$ production. Epo-induced erythropoiesis is concomitant with autophagy flux induction. TNF $\alpha$ binds its receptor TNFR to activate neutral sphingomyelinase (nSMase) catalyzing ceramide (Cer) production. Ceramides inhibit erythroid gene expression and induce GATA-2, PU.1, CD11b, and miR-155, -146a, and -223 expression. $\mathrm{TNF} \alpha$ inhibits SphK1 leading to a ceramide/S1P unbalanced reaction. $\mathrm{S} 1 \mathrm{P}$ has a positive effect on $\mathrm{Hb}$ production and erythroid marker

only TNF $\alpha$ induced miR-146a and miR-155. In agreement with our results, these miRs are also inhibitors of erythropoiesis [13, 48-50].

Autophagy is required for physiological erythropoiesis to eliminate organelles. Knock out of the autophagic regulators, ULK1, Atg4, Atg7, or FIP200, or mitophagyspecific receptor, NIX, inhibited erythropoiesis leading to anemia in mice while inducing myeloid expansion $[16,18$, 51-53]. In contrast to erythropoiesis, autophagy is not essential for neutrophil differentiation [17]. At $24 \mathrm{~h}$, Beclin1 induction, Atg5/Atg 12 complex formation, the presence of GFP-LC3 punctae but the absence of mature autophagosomes, and sustained p62/SQSTM1 protein expression suggested that autophagy was initiated but not completed in EpoCD34/HSPCs. Nevertheless, this early autophagic flux was inhibited by TNF $\alpha$ and bSMase. Western blot analysis showed that TNF $\alpha$ and GW4869 did not change endogenous LC3-II levels. Besides its presence in autophagic membranes, LC3-II can be generated in an autophagyindependent manner and a significant amount of LC3-II was detectable after autophagy inhibition [54]. At $96 \mathrm{~h}$, EpoCD34/HSPCs presented autophagic features, while $\mathrm{TNF} \alpha$ and bSMase inhibited autophagy. At the molecular expression. TNF $\alpha$ and ceramides inhibit autophagy. TNF $\alpha$ phosphorylates mTOR (P-mTOR) via the PI3K/AKT pathway, while ceramide activates mTOR through a different mechanism. P-mTOR leads to ULK1 inhibition and the subsequent Atg13 dephosphorylation. TNF $\alpha$ and ceramides inhibit Beclin-1 (BCN1) expression, the formation of the Atg5-Atg12 complex, GFP-LC3 punctae, and autophagosomes, and induce p62/SQSTM1 accumulation. Together, the effects triggered by the TNF $\alpha /$ neutral SMase/ceramide pathway at the molecular level lead to autophagy and erythropoiesis inhibition and myelopoiesis induction

level, the $\mathrm{PI} 3 \mathrm{~K}\left(\mathrm{p}^{2} 5^{\mathrm{Y} 458}\right) / \mathrm{AKT}^{\mathrm{S} 473} / \mathrm{mTOR}^{\mathrm{S} 2448} / \mathrm{ULK}^{\mathrm{S} 758}$ pathway was activated by $\mathrm{TNF} \alpha$. Interestingly, bSMase activated the $\mathrm{mTOR}^{\mathrm{S} 2448} / \mathrm{ULK}^{\mathrm{S} 758}$ pathway independently of the PI3K(p85 $\left.{ }^{\mathrm{Y} 458}\right) / \mathrm{AKT}^{\mathrm{S} 473}$ pathway. This was supported by the fact that GW4869 was unable to prevent TNFoinduced PI3K/AKT activation. In agreement with our results, autophagy was inhibited in TNF $\alpha$-treated Caco-2 cells, and TNF $\alpha$ acted as an activator of mTOR via IкBkinases involving or not AKT in different cancer cell models $[55,56] . \operatorname{Atg} 13^{\mathrm{S} 355}$ phosphorylation was inhibited by TNF $\alpha$ and bSMase, in agreement with ULK1 inactivation, supporting an inhibitory effect on autophagy induction. Our results also indicate that ceramides and TNF $\alpha$ may affect autophagy through distinct mechanisms in EpoCD34/ HSPCs. Another evidence of autophagy inhibition was the expression of Rubicon after $96 \mathrm{~h}$ of TNF $\alpha$ treatment while it was absent in untreated EpoCD34/HSPCs. Rubicon suppresses autophagosome maturation through physical interaction between its RUN domain and the catalytic subunit of $\mathrm{hVps34}$. Indeed, ectopically expressed Rubicon reduced autophagosome maturation in U2OS cells, while Rubicon depletion accelerated p62/SQSTM1 protein degradation [21]. Our results also showed that $\mathrm{TNF} \alpha$ and bSMase 
induced an accumulation of p62/SQSTM1 in EpoCD34/ HSPCs after $96 \mathrm{~h}$ in correlation with autophagy inhibition [57]. Our results obtained by TEM, GFP-LC3 transfection, investigation of signaling pathways, and autophagy protein expression levels demonstrated that $\mathrm{TNF} \alpha$ and ceramides affect autophagy in EpoCD34/HSPCs. The inhibition of early and late steps of autophagy by si-Atg5 and BafA1 treatment, respectively, led to inhibition of erythropoiesis of EpoCD34/HSPCs comparable to a treatment by TNF $\alpha$ and bSMase. Accordingly, the mTOR inhibitor rapamycin was able to prevent the effect of TNF $\alpha$ and bSMase by inducing both autophagy and erythropoiesis.

Our results demonstrate that the impairment of erythropoiesis results from an inhibition of autophagy in TNF $\alpha$ - and ceramide-treated CD34/HSPCs. Granulocytic differentiation was prevented by rapamycin in $\mathrm{TNF} \alpha$-treated cells, while the late-step inhibitor of autophagy BafA1 induced differentiation underlining the role of autophagy inhibition in this differentiation pathway. Nevertheless, Atg5 knockdown was not sufficient to induce granulocytic differentiation in the presence of Epo, suggesting that erythropoiesis impairment is not the direct cause of myeloid differentiation. Altogether, these results underline the key role of autophagy via mTOR signaling in the modulation of EpoCD34/HSPC differentiation by TNF $\alpha$, nSMase, and ceramides.

Overall, our results demonstrate that the TNF $\alpha / n S M a s e /$ ceramide pathway interferes with hematopoietic homeostasis providing new insights into hematopoietic cell fate determination as well as in inflammation-related anemia. Inhibition of erythropoiesis and induction of myelopoiesis

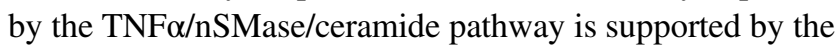
modulation of key regulators of hematopoiesis. Furthermore, autophagy inhibition is a key event in impairment of erythropoiesis and induction of granulocytic differentiation in EpoCD34/HSPCs.

\section{Materials and methods}

\section{Antibodies and reagents}

Desmethylimipramine (desipramine), $\quad N, N^{\prime}$-Bis[4-(4,5dihydro-1H-imidazol-2-yl)phenyl]-3,3'-p-phenylene-bisacrylamide dihydrochloride (GW4869), SMase from Staphylococcus aureus, $\mathrm{C} 2$ dihydroceramide, S1P, and BafA1 were purchased from Sigma-Aldrich (Bornem, Belgium), C2-ceramide from Santa Cruz Biotechnology (Boechout, Belgium) and C6-ceramide from Enzo Life Sciences (Antwerp, Belgium). Human recombinant TNF $\alpha$ was kindly provided by Pr. Athanase Visvikis (Université de Lorraine, Nancy, France). Stem cell factor (SCF) and interleukin (IL)3 were purchased from ReliaTech (Wolfenbüttel,
Germany). The erythroid differentiation is induced with human recombinant erythropoietin (Epoietin beta, Neorecormon Roche, Grenzach-Whylen, Germany). The primary antibodies used were directed against: GATA-1, GATA-2, PU.1, $\alpha$-, $\beta$-, and $\gamma$-globin (Santa Cruz Biotechnology), SphK1, PI3K, P-PI3K ${ }^{\mathrm{Y} 458}$, AKT, P-AKT ${ }^{\mathrm{S} 473}$,

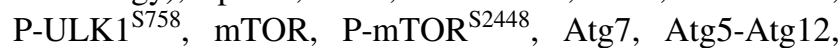
Atg13, P-Atg13 ${ }^{\mathrm{S} 355}$, Beclin-1, p62/SQSTM1 (Cell Signaling, Leiden, The Netherlands), LC3 (Sigma), Rubicon (Abcam, Cambridge, UK), and ceramide MID 15B4 (Enzo Life Sciences, Antwerpen, Belgium), and the antibodies used for flow cytometry (CD235a/GPA, CD11b) were purchased from BD BioSciences (Erembodegem, Belgium).

\section{$\mathrm{CD}^{+}{ }^{+} / \mathrm{HSPC}$ isolation, culture, and treatment}

UCB is kindly provided by Clinique Bohler, Luxembourg and Unité de Thérapie Cellulaire et banque de Tissus (UTCT), Nancy, France. It is collected after normal deliveries with a donor's written informed consent in agreement with the National Committee of Research Ethics in Luxembourg. $\mathrm{CD} 34^{+} / \mathrm{HSPCs}$ were purified as previously described $[2,58]$. Briefly, mononuclear cells were collected using Ficoll ${ }^{\circ}$ (GE Healthcare, Roosendaal, The Netherlands) density gradient medium. Then, $\mathrm{CD} 34^{+}$cells were selected by magnetic cell sorting following the manufacturer's instructions (Miltenyi Biotec, Utrecht, The Netherlands). $\mathrm{CD}_{3}{ }^{+}$cells obtained were then cultivated in serum-free condition in Stem Line Medium II (Sigma) supplemented with L-glutamine, penicillin/streptomycin/amphotericin B (Lonza, Verviers, Belgium), IL-3 $(10 \mathrm{ng} / \mathrm{mL})$, and SCF $(50 \mathrm{ng} / \mathrm{mL})$ for 3 days (Supplementary Figure S10). At days 0,4 , and 6 , epoietin beta (Epo) $(2 \mathrm{U} / \mathrm{mL})$ was added $1 \mathrm{~h}$ after the additon of TNF $\alpha(20 \mathrm{ng} / \mathrm{mL}), \mathrm{C} 2$-ceramide $(30 \mu \mathrm{M}), \mathrm{C} 2$ dihydroceramide $(30 \mu \mathrm{M})$, or the bSMase $(0.5 \mathrm{U} / \mathrm{mL})$. The Smase inhibitors GW4869 $(20 \mu \mathrm{M})$ and desipramine $(10 \mu \mathrm{M})$ were added $1 \mathrm{~h}$ before TNF $\alpha$ treatment. Cultures were maintained at $2 \times 10^{5}$ cells $/ \mathrm{mL}$. IL-3 was removed from the medium on day 4 and SCF on day 6 . Cell number and viability were assessed using Trypan blue dye exclusion assay, and all cells were cultured in an incubator at $37^{\circ} \mathrm{C}$, $95 \%$ humidity, and $5 \% \mathrm{CO}_{2}$. Cell viability was confirmed by Annexin V/propidium iodide staining and fluorescenceactivated cell sorter (FACS) analysis according to the manufacturer's protocol (BD Biosciences).

\section{Profiling of ceramides by ESI-MS/MS}

Ceramides were extracted according to the method by Kyrklund [59] in the presence of deuterium-labeled standards ( $N$-heptadecanoyl-D-erythro-sphingosine (C17:0-ceramide); $\mathrm{N}$-palmitoyl (d31)-D-erythro-sphingosylphosphorylcholine (C16:0D31SM) from Avanti Polar Lipids, Alabama, USA). Briefly, total lipids 
were extracted in chloroform:methanol (1:2 (v/v)). Sphingolipids were isolated by saponification after the addition of internal standards, and fractionated and desalted using reverse-phase Bond Elut $\mathrm{C} 18$ columns. The dry extracts were kept at $-20^{\circ} \mathrm{C}$ until MS/MS analysis. Samples were homogenized in chloroform:methanol $(1: 2(\mathrm{v} / \mathrm{v}))$ and analyzed by direct flow injection on a triple-quadrupole mass spectrometer (API 4500 QTRAP MS/MS; Sciex Applied Biosystems, Toronto, ON, Canada) in the positive ionization mode using the multiple reaction monitoring (MRM) method. Ceramide species were measured with a flow rate of $200 \mu \mathrm{l} / \mathrm{min}$ (analysis time of $3 \mathrm{~min}$ ). The quantity of each molecular species was calculated from the ratio of its signal to that of the corresponding internal standard and normalized to the amount of cells.

\section{Assessment of cell differentiation}

Erythroid differentiation was assessed by evaluating the rate of hemoglobin-producing cells after benzidine staining as previously described [2]. Granulocytic differentiation was assessed by evaluating the rate of formazan depositproducing cells upon activation with PMA (phorbol 12myristate 13-acetate) by NBT assay. Differentiation status of $\mathrm{CD} 34^{+}$cells was determined by microscopy analysis of the cell morphology (Leica DM2000 microscope, Lecuit, Howald, Luxembourg) at day 9 after MGG staining (Merck, Leuven, Belgium) and by flow cytometry analysis (FACSCalibur, Beckton-Dickinson, San Jose, CA, USA) of CD235a/GPA erythroid markers and CD11b granulomonocytic markers at days $0,4,6$, and 9 . Results were analyzed using the FlowJo software (version 8.8.7, Tree Star, Ashland, OR, USA) and statistical analysis was based on 10,000 events per sample. Colony formation assay was performed by seeding $\mathrm{CD} 34^{+}$cells in Methocult $\mathrm{H} 4230$ (StemCell Technologies, Vancouver, BC, Canada) supplemented with L-glutamine, penicillin/streptomycin/amphotericin B (Lonza), IL-3 $(10 \mathrm{ng} / \mathrm{mL})$, and SCF $(50 \mathrm{ng} / \mathrm{mL})$ and treated in the same conditions than suspension cultures. Colonies were counted after 14 days according to featured differentiation types and images were collected with a Leica DMIRB microscope.

\section{CD34/HSPC transfection}

CD34/HSPCs were transfected with nSMase 2 (smpd3) predesigned siRNA (Thermofisher, Erembodegen, Belgium) and Atg5 siRNA (Qiagen, Venlo, The Netherlands). Cells were transfected using the Human CD34 cell Nucleofector Kit (Lonza, Westburg, Leusden, The Netherlands). Transfection efficiency was assessed by Western blot and realtime PCR analysis of nSMase 2 and Atg5 expression at $24 \mathrm{~h}$.

CD34/HSPCs were transfected at $0 \mathrm{~h}$ with pSELECTGFP-hLC3 plasmid (Invivogen, Toulouse, France) using the Human CD34 cell Nucleofector ${ }^{\oplus}$ Kit according to the manufacturer's instructions. After $4 \mathrm{~h}$, cells were treated, and the transfection efficiency of GFP-LC3, as well as the effect of the different treatments on punctae formation, were assessed by fluorescence microscopy after 16 and $24 \mathrm{~h}$ (Olympus, Aartselaar, Belgium). Nuclei were stained with 4',6-diamidino-2-phenylindole (DAPI).

\section{Transmission electron microscopy}

Cell samples were fixed for $1 \mathrm{~h}$ in $2.5 \%$ glutaraldehyde (Euromedex, Mundolsheim, France), diluted in $0.1 \mathrm{M}$ cacodylate buffer (Euromedex), pH 7.2, and washed twice in cacodylate buffer. Cells were then postfixed $2 \mathrm{~h}$ in $2 \%$ osmium tetraoxide, washed with distilled water, and stained with $0.5 \%$ uranyl acetate overnight. Samples were then dehydrated in successive ethanol washes, rinsed in propylene oxide, and embedded overnight in 1:1 propylene oxide/ Spurr's resin and left to polymerize at $56^{\circ} \mathrm{C}$ for $48 \mathrm{~h}$. Ultrathin sections of $70-90 \mathrm{~nm}$ were cut with an ultramicrotome, EM UC7 (Leica), stained with uranyl acetate plus lead citrate, and viewed using a JEM1010 transmission electron microscope (JEOL, Japan).

\section{Reverse transcription and real-time PCR}

Total RNAs were extracted and quantified as previously described [60]. One microgram of isolated RNA was reverse transcribed according to the manufacturer's protocol using the miScript II RT Kit (Qiagen) for miR analysis and the SuperScript ${ }^{\mathrm{TM}}$ III Reverse Transcriptase Kit (Invitrogen, Tournai, Belgium) for mRNA. Real-time PCR was performed using 7300 Real-Time PCR System (Applied Biosystems, Halle, Belgium). Specific forward and reverse primers or references are described in Supplementary Tables S3 and S4. Relative expression levels were determined using the $2^{-\Delta \Delta \mathrm{Ct}}$ method. Internal standard genes RNUIA and $\beta$-actin were used as endogenous quantity control to normalize microRNA and mRNA levels, respectively.

\section{Western blotting}

Total cell lysates were performed using the M-PER ${ }^{\bullet}$ reagent (Thermo Fisher) and nuclear/cytoplasmic proteins were extracted as previously described [60]. Buffers for proteins extraction contained anti-protease and antiphosphatase cocktail. Protein extracts were separated by sodium dodecyl sulfate-polyacrylamide gel electrophoresis and then transferred to PVDF membrane. Unspecific binding sites were blocked by incubating membranes in phosphate-buffered saline containing $0.5 \%$ Tween-20 and $5 \%$ milk or bovine serum albumin. Immunoblots were 
incubated with a specific primary antibody and a horse radish peroxidase-conjugated secondary antibody. Blots were developed using an enhanced chemiluminescence method ( $\mathrm{ECL}^{+} / \mathrm{Amersham}$ ), and luminescent signal was analyzed with the ImageQuant Las 4000 Mini software (GE Healthcare). Western blot quantifications were performed using the ImageJ64 software (Supplementary Figure S11).

\section{Statistical analysis}

Statistical analysis was performed using the Prism 7 software (GraphPad software, La Jolla, CA, USA). Data correspond to mean $\pm \mathrm{SD}$. Results were analyzed using paired $T$-test, one-way, two-way ANOVA (analysis of variance) followed by post hoc tests (see legends). $P$ values were considered statistically significant when $p<0.05$.

Acknowledgements We thank the staff of the Clinique Bohler (Luxembourg) and the "Unité de Thérapie Cellulaire et Banque de Tissus" (UTCT), Nancy (France) for collecting umbilical cord blood as well as $\operatorname{Pr}$ A. Visvikis (Université de Lorraine, Nancy, France) for providing TNF $\alpha$. MO, SC, and AG were supported by "Télévie - Luxembourg" grants. JR was supported by grants from "Recherches Scientifiques Luxembourg" and Brain Korea (BK21) PLUS program. Research at LBMCC was supported by the "Recherche Cancer et Sang" foundation, the "Recherches Scientifiques Luxembourg" association, by the "En Häerz fir kriibskrank Kanner" association, by the Action LIONS "Vaincre le Cancer" association, and by Télévie Luxembourg. MD thanks the Research Institute of Pharmaceutical Sciences, College of Pharmacy, Seoul National University, South Korea; the Tumor Microenvironment GCRC (2011-0030001) from the National Research Foundation funded by the Ministry of Science and ICT of Korea; the Creative-Pioneering Researchers Program through Seoul National University (funding number: 370C-20160062) and the Brain Korea (BK) 21 Plus program, Korea.

\section{Compliance with ethical standards}

Conflict of interest The authors declare that they have no conflict of interest.

Open Access This article is licensed under a Creative Commons Attribution 4.0 International License, which permits use, sharing, adaptation, distribution and reproduction in any medium or format, as long as you give appropriate credit to the original author(s) and the source, provide a link to the Creative Commons license, and indicate if changes were made. The images or other third party material in this article are included in the article's Creative Commons license, unless indicated otherwise in a credit line to the material. If material is not included in the article's Creative Commons license and your intended use is not permitted by statutory regulation or exceeds the permitted use, you will need to obtain permission directly from the copyright holder. To view a copy of this license, visit http://creativecommons. org/licenses/by/4.0/.

\section{References}

1. Spivak JL. The anaemia of cancer: death by a thousand cuts. Nat Rev Cancer. 2005;5:543-55.
2. Grigorakaki C, Morceau F, Chateauvieux S, Dicato M, Diederich M. Tumor necrosis factor alpha-mediated inhibition of erythropoiesis involves GATA-1/GATA-2 balance impairment and PU.1 over-expression. Biochem Pharmacol. 2011;82:156-66.

3. Buck I, Morceau F, Cristofanon S, Reuter S, Dicato M, Diederich M. The inhibitory effect of the proinflammatory cytokine TNFalpha on erythroid differentiation involves erythroid transcription factor modulation. Int J Oncol. 2009;34: 853-60.

4. Buck I, Morceau F, Cristofanon S, Heintz C, Chateauvieux S, Reuter $\mathrm{S}$, et al. Tumor necrosis factor alpha inhibits erythroid differentiation in human erythropoietin-dependent cells involving p38 MAPK pathway, GATA-1 and FOG-1 downregulation and GATA-2 upregulation. Biochem Pharmacol. 2008; 76:1229-39.

5. Morceau F, Schnekenburger M, Blasius R, Buck I, Dicato M, Diederich M. Tumor necrosis factor alpha inhibits aclacinomycin A-induced erythroid differentiation of K562 cells via GATA-1. Cancer Lett. 2006;240:203-12.

6. Wickrema A, Crispino JD. Erythroid and megakaryocytic transformation. Oncogene. 2007;26:6803-15.

7. Mathieu J, Ruohola-Baker H. Regulation of stem cell populations by microRNAs. Adv Exp Med Biol. 2013;786:329-51.

8. Ikonomi P, Rivera CE, Riordan M, Washington G, Schechter AN, Noguchi CT. Overexpression of GATA-2 inhibits erythroid and promotes megakaryocyte differentiation. Exp Hematol. 2000;28: 1423-31.

9. Dore LC, Amigo JD, Dos Santos CO, Zhang Z, Gai X, Tobias JW, et al. A GATA-1-regulated microRNA locus essential for erythropoiesis. Proc Natl Acad Sci USA. 2008;105:3333-8.

10. Zhang P, Zhang X, Iwama A, Yu C, Smith KA, Mueller BU, et al. PU.1 inhibits GATA-1 function and erythroid differentiation by blocking GATA-1 DNA binding. Blood. 2000;96:2641-8.

11. Fukao T, Fukuda Y, Kiga K, Sharif J, Hino K, Enomoto Y, et al. An evolutionarily conserved mechanism for microRNA-223 expression revealed by microRNA gene profiling. Cell. 2007;129: 617-31.

12. Ghani S, Riemke P, Schonheit J, Lenze D, Stumm J, Hoogenkamp $\mathrm{M}$, et al. Macrophage development from HSCs requires PU.1coordinated microRNA expression. Blood. 2011;118:2275-84.

13. Squadrito ML, Etzrodt M, De Palma M, Pittet MJ. MicroRNAmediated control of macrophages and its implications for cancer. Trends Immunol. 2013;34:350-9.

14. Betin VM, Singleton BK, Parsons SF, Anstee DJ, Lane JD. Autophagy facilitates organelle clearance during differentiation of human erythroblasts: evidence for a role for ATG4 paralogs during autophagosome maturation. Autophagy. 2013;9:881-93.

15. Kang YA, Sanalkumar R, O'Geen H, Linnemann AK, Chang CJ, Bouhassira EE, et al. Autophagy driven by a master regulator of hematopoiesis. Mol Cell Biol. 2012;32:226-39.

16. Mortensen M, Ferguson DJ, Edelmann M, Kessler B, Morten KJ, Komatsu M, et al. Loss of autophagy in erythroid cells leads to defective removal of mitochondria and severe anemia in vivo. Proc Natl Acad Sci USA. 2010;107:832-7.

17. Rozman S, Yousefi S, Oberson K, Kaufmann T, Benarafa C, Simon HU. The generation of neutrophils in the bone marrow is controlled by autophagy. Cell Death Differ. 2015;22:445-56.

18. Zhang J, Wu K, Xiao X, Liao J, Hu Q, Chen H, et al. Autophagy as a regulatory component of erythropoiesis. Int $\mathrm{J}$ Mol Sci. 2015;16:4083-94.

19. Papinski D, Kraft C. Regulation of autophagy by signaling through the Atg1/ULK1 complex. J Mol Biol. 2016;428 (9 Part A):1725-41.

20. Kim J, Kundu M, Viollet B, Guan KL. AMPK and mTOR regulate autophagy through direct phosphorylation of Ulk1. Nat Cell Biol. 2011;13:132-41. 
21. Sun Q, Zhang J, Fan W, Wong KN, Ding X, Chen S, et al. The RUN domain of rubicon is important for hVps34 binding, lipid kinase inhibition, and autophagy suppression. J Biol Chem. 2011;286:185-91.

22. Mizushima N, Yoshimori T, Ohsumi Y. The role of Atg proteins in autophagosome formation. Annu Rev Cell Dev Biol. 2011;27: 107-32.

23. Pankiv S, Clausen TH, Lamark T, Brech A, Bruun JA, Outzen H, et al. p62/SQSTM1 binds directly to Atg8/LC3 to facilitate degradation of ubiquitinated protein aggregates by autophagy. $\mathbf{J}$ Biol Chem. 2007;282:24131-45.

24. Liu JJ, Hou SC, Shen CK. Erythroid gene suppression by NFkappa B. J Biol Chem. 2003;278:19534-40.

25. Schneider-Brachert W, Tchikov V, Neumeyer J, Jakob M, Winoto-Morbach S, Held-Feindt J, et al. Compartmentalization of TNF receptor 1 signaling: internalized TNF receptosomes as death signaling vesicles. Immunity. 2004;21:415-28.

26. Yang Z, Costanzo M, Golde DW, Kolesnick RN. Tumor necrosis factor activation of the sphingomyelin pathway signals nuclear factor kappa B translocation in intact HL-60 cells. J Biol Chem. 1993;268:20520-3.

27. Dressler KA, Mathias S, Kolesnick RN. Tumor necrosis factoralpha activates the sphingomyelin signal transduction pathway in a cell-free system. Science. 1992;255:1715-8.

28. Adam-Klages S, Adam D, Wiegmann K, Struve S, Kolanus W, Schneider-Mergener J, et al. FAN, a novel WD-repeat protein, couples the p55 TNF-receptor to neutral sphingomyelinase. Cell. 1996;86:937-47.

29. Maceyka M, Spiegel S. Sphingolipid metabolites in inflammatory disease. Nature. 2014;510:58-67.

30. Harvald EB, Olsen AS, Faergeman NJ. Autophagy in the light of sphingolipid metabolism. Apoptosis. 2015;20:658-70.

31. Newton J, Lima S, Maceyka M, Spiegel S. Revisiting the sphingolipid rheostat: Evolving concepts in cancer therapy. Exp Cell Res. 2015;333:195-200.

32. Dallalio G, North M, Worden BD, Means RT Jr. Inhibition of human erythroid colony formation by ceramide. Exp Hematol. 1999;27:1133-8.

33. Muntean AG, Crispino JD. Differential requirements for the activation domain and FOG-interaction surface of GATA-1 in megakaryocyte gene expression and development. Blood. 2005; 106:1223-31.

34. Airola MV, Allen WJ, Pulkoski-Gross MJ, Obeid LM, Rizzo RC, Hannun YA. Structural basis for ceramide recognition and hydrolysis by human neutral ceramidase. Structure. 2015;23:1482-91.

35. Okazaki T, Bell RM, Hannun YA. Sphingomyelin turnover induced by vitamin D3 in HL-60 cells. Role in cell differentiation. J Biol Chem. 1989;264:19076-80.

36. Kim MY, Linardic C, Obeid L, Hannun Y. Identification of sphingomyelin turnover as an effector mechanism for the action of tumor necrosis factor alpha and gamma-interferon. Specific role in cell differentiation. J Biol Chem. 1991;266:484-9.

37. Buitenhuis M, Verhagen LP, van Deutekom HW, Castor A, Verploegen S, Koenderman L, et al. Protein kinase B (c-akt) regulates hematopoietic lineage choice decisions during myelopoiesis. Blood. 2008;111:112-21.

38. Souza LR, Silva E, Calloway E, Cabrera C, McLemore ML. GCSF activation of AKT is not sufficient to prolong neutrophil survival. J Leukoc Biol. 2013;93:883-93.

39. Martelli AM, Evangelisti C, Chiarini F, Grimaldi C, Cappellini A, Ognibene A, et al. The emerging role of the phosphatidylinositol 3-kinase/Akt/mammalian target of rapamycin signaling network in normal myelopoiesis and leukemogenesis. Biochim Biophys Acta. 2010;1803:991-1002.
40. Trocoli A, Bensadoun P, Richard E, Labrunie G, Merhi F, Schlafli AM, et al. p62/SQSTM1 upregulation constitutes a survival mechanism that occurs during granulocytic differentiation of acute myeloid leukemia cells. Cell Death Differ. 2014;21:1852-61.

41. Takemoto CM, Brandal S, Jegga AG, Lee YN, Shahlaee A, Ying $\mathrm{Y}$, et al. PU.1 positively regulates GATA-1 expression in mast cells. J Immunol. 2010;184:4349-61.

42. Ohneda K, Yamamoto M. Roles of hematopoietic transcription factors GATA-1 and GATA-2 in the development of red blood cell lineage. Acta Haematol. 2002;108:237-45.

43. Rhodes J, Hagen A, Hsu K, Deng M, Liu TX, Look AT, et al. Interplay of pu. 1 and gata1 determines myelo-erythroid progenitor cell fate in zebrafish. Dev Cell. 2005;8:97-108.

44. Whyatt D, Lindeboom F, Karis A, Ferreira R, Milot E, Hendriks $\mathrm{R}$, et al. An intrinsic but cell-nonautonomous defect in GATA-1overexpressing mouse erythroid cells. Nature. 2000;406:519-24.

45. Ikonomi P, Noguchi CT, Miller W, Kassahun H, Hardison R, Schechter AN. Levels of GATA-1/GATA-2 transcription factors modulate expression of embryonic and fetal hemoglobins. Gene. 2000;261:277-87.

46. Stopka T, Amanatullah DF, Papetti M, Skoultchi AI. PU.1 inhibits the erythroid program by binding to GATA-1 on DNA and creating a repressive chromatin structure. EMBO J. 2005;24: 3712-23.

47. Yamada T, Abe M, Higashi T, Yamamoto H, Kihara-Negishi F, Sakurai T, et al. Lineage switch induced by overexpression of Ets family transcription factor PU.1 in murine erythroleukemia cells. Blood. 2001;97:2300-7.

48. Felli N, Pedini F, Romania P, Biffoni M, Morsilli O, Castelli G, et al. MicroRNA 223-dependent expression of LMO2 regulates normal erythropoiesis. Haematologica. 2009;94:479-86.

49. Starczynowski DT, Kuchenbauer F, Wegrzyn J, Rouhi A, Petriv O, Hansen CL, et al. MicroRNA-146a disrupts hematopoietic differentiation and survival. Exp Hematol. 2011;39: $167-78$ e 164.

50. Masaki S, Ohtsuka R, Abe Y, Muta K, Umemura T. Expression patterns of microRNAs 155 and 451 during normal human erythropoiesis. Biochem Biophys Res Commun. 2007;364: 509-14.

51. Zhang J, Randall MS, Loyd MR, Dorsey FC, Kundu M, Cleveland JL, et al. Mitochondrial clearance is regulated by Atg7dependent and -independent mechanisms during reticulocyte maturation. Blood. 2009;114:157-64.

52. Kundu M, Lindsten T, Yang CY, Wu J, Zhao F, Zhang J, et al. Ulk1 plays a critical role in the autophagic clearance of mitochondria and ribosomes during reticulocyte maturation. Blood. 2008;112:1493-502.

53. Sandoval H, Thiagarajan P, Dasgupta SK, Schumacher A, Prchal JT, Chen M, et al. Essential role for Nix in autophagic maturation of erythroid cells. Nature. 2008;454:232-5.

54. Mizushima N, Yoshimori T, Levine B. Methods in mammalian autophagy research. Cell. 2010;140:313-26.

55. Dan HC, Baldwin AS. Differential involvement of IkappaB kinases alpha and beta in cytokine- and insulin-induced mammalian target of rapamycin activation determined by Akt. J Immunol. 2008;180:7582-9.

56. Zhang C, Yan J, Xiao Y, Shen Y, Wang J, Ge W, et al. Inhibition of autophagic degradation process contributes to claudin-2 expression increase and epithelial tight junction dysfunction in TNF-alpha treated cell monolayers. Int J Mol Sci. 2017;18. pii: E157

57. Katsuragi Y, Ichimura Y, Komatsu M. p62/SQSTM1 functions as a signaling hub and an autophagy adaptor. FEBS J. 2015;282: 4672-8. 
58. Carlile GW, Smith DH, Wiedmann M. Caspase-3 has a nonapoptotic function in erythroid maturation. Blood. 2004;103:4310-6.

59. Kyrklund T. Two procedures to remove polar contaminants from a crude brain lipid extract by using prepacked reversed-phase columns. Lipids. 1987;22:274-7.
60. Trecul A, Morceau F, Gaigneaux A, Schnekenburger M, Dicato M, Diederich M. Valproic acid regulates erythro-megakaryocytic differentiation through the modulation of transcription factors and microRNA regulatory micro-networks. Biochem Pharmacol. 2014;92:299-311. 